Jational Bureau of Standards

$\sim$ Library, N.W. Bldg

\title{
JAN 111961
}

\section{Reference book not to be taken from the niiviary.}

PART B

SOLAR - GEOPHYSICAL DATA

ISSUED

DECEMBER 1960

U. S. DEPARTMENT OF COMMERCE

NATIONAL BUREAU OF STANDARDS

CENTRAL RADIO PROPAGATION LABORATORY

BOULDER, COLORADO 



\section{SOLAR - GEOPHYSICAL DATA}

\section{CONTENTS}

\section{DAILY SOLAR INDICES}

(a) Relative Sunspot Numbers and 2800 .Wc Solar F1ux

(b) Graph of Sunspot Cycle

\section{SOLAR CENTERS OF ACTIVITY}

(a) Calcium Plage and Sunspot Regions

(b-c) Provisional Coronal Line Emission Indices - 0ctober, November 1960

$(d-f)$ Final Coronal Line Emission Indices - July, August, September 1960

III SOLAR FLARES

(a-e) Uptical Observations - November 1960

(f) Flare Patrol Observations - November 1960

$(g-h)$ Subflares - october 1960

(i-n) Optical Observations - August 1960

(o) Flare Patrol Ubservations - August 1960

(p) Optical Observations - February 1960

(q) Ionospheric Effects (SWF) - October 1960

(r) Ionospheric Effects (SEA-SCNA-Bursts) October 1960

IV SOLAR RADIO WAVES

(a) 2800 ic - Outstanding Occurrences (Ottawa) November 1960

(b) 169 Mc - Outstanding Occurrences (Nancay) November 1960

(c-d) $108 \mathrm{Mc}$ - Outstanding Occurrences (Boulder) November 1960

(e-i) $9.1 \mathrm{~cm}$ - Spectroheliograms (Stanford) April 1960

\section{$\mathrm{V}$ COSMIC RAY INDICES}

(a) Climax Neutron Monitor - October 1960

(b) Deep River Neutron Monitor - October 1960

VI GEOMAGNETIC ACTIVITY INDICES

(a) C, Kp, Ap, and Selected Quiet and Disturbed Days

(b) Chart of Kp by Solar Rotations

\section{RADIO PROPAGATION QUALITY INDICES}

North At lantic:

(a) CRPL Quality Figures and Forecasts

(b) Graphs Comparing Forecast and Observed Quality

(c-d) Graphs of Useful Frequency Ranges

North Pac if ic:

(e) CRPL Quality Figures and Forecasts

(f) Graphs Comparing Forecast and Observed Quality

VIII ALERT PERIODS AND SPECIAL WORLD INTERVALS

(a) 1960 Alerts and SWI 

$9.1 \mathrm{~cm}$ Spectroheliograms

The microwave spectroheliograms obtained at the Radioscience Laboratory of the Stanford University, Stanford, California (N $37^{\circ} 24^{\prime}$, ' $122^{\circ} 11^{\prime}$ ) are presented.

The maps show the disk distribution of solar radio emission at a wavelength of $9.1 \mathrm{~cm}$ by means of radioisophotes, or lines of constant brightness temperature. The contour interval, which varies from map to map, is usually about $30,000^{\circ} \mathrm{K}$, and is determined after the map is drawn by reference to the measured flux density of the whole sun. A circle shows the photosphere; a correction has been applied for the variation of the sun's semi-diameter, so that the photospheric circle is reproduced with a constant diameter of $5 \mathrm{~cm}$. This is an integral submultiple of the IAU standard of $15 \mathrm{~cm}$ used on the full size originals, which are available as Stanford Badio Astronomy Institute Publications.

A full description of the Stanford microwave spectroheliograph has been given by Bracewell and Swarup (IIBE Trans., Vol. AP 9, January 1961). With this instrument the sun is scanned along approximately fifteen parallel lines from west to east, and from the resulting records the maps are carefully prepared by hand. A positional accuracy of better than \pm 1 minute of arc in the location of bright features is maintained.

At the zenith, the beam of the instrument is circular with a beamwidth of 3.1 minutes of arc. At a declination son the merian the beamwidths are 3.1 minutes east-west and $3.1 \mathrm{sec}\left(30^{\circ} 2-8\right)$ north-south. To illustrate the magnitude and position of the subsidary lobes surrounding the beam, the theoretical response to a point solurce is given below.

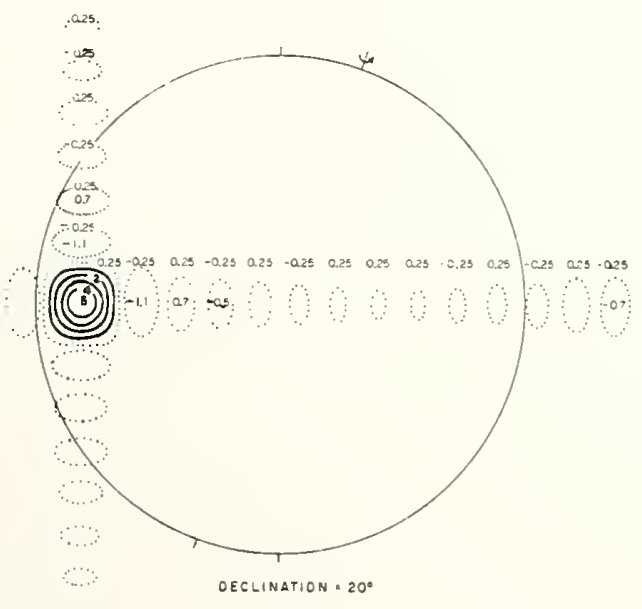


Further details are given in the above mentioned reference. The response to a source of finite size is the convolution of this pattern with the source distribution. The subsidary lobes may be reduced by smoothing the maps but this would also widen the primary beam. A simple procedure for smoothing is to average four adjacent values situated at the corner of a rectangle with sides parallel to the scanning direction and 0.8 beamwidths long $(R$. N. Bracewell, "Correcting no ise maps for beamwidth", Radio No ise Spectrum, ed. D. II. Menzel, pp. 141-150, Harvard 1960). 
The descriptive text was published separately, November 1960. 


\begin{tabular}{|c|c|}
\hline $\begin{array}{l}\text { Oct. } \\
1960\end{array}$ & $\begin{array}{c}\text { American Relative } \\
\text { Sunspot Numbers } \\
\text { RA }_{\mathrm{A}^{\prime}}\end{array}$ \\
\hline 1 & 11 \\
\hline 2 & 24 \\
\hline 3 & 20 \\
\hline 4 & 43 \\
\hline 5 & 75 \\
\hline 6 & 73 \\
\hline 7 & 112 \\
\hline 8 & 94 \\
\hline 9 & 138 \\
\hline 10 & 143 \\
\hline 11 & 103 \\
\hline 12 & 119 \\
\hline 13 & 98 \\
\hline 14 & 93 \\
\hline 15 & 96 \\
\hline 16 & 91 \\
\hline 17 & 88 \\
\hline 18 & 68 \\
\hline 19 & 68 \\
\hline 20 & 70 \\
\hline 21 & 63 \\
\hline 22 & 47 \\
\hline 23 & 47 \\
\hline 24 & 52 \\
\hline 25 & 53 \\
\hline 26 & 53 \\
\hline 27 & 57 \\
\hline 28 & 48 \\
\hline 29 & 70 \\
\hline 30 & 64 \\
\hline 31 & 72 \\
\hline Mean: & 72.7 \\
\hline
\end{tabular}

\begin{tabular}{|c|c|c|}
\hline $\begin{array}{l}\text { Nov. } \\
1960\end{array}$ & $\begin{array}{c}\text { Zürich Provisional } \\
\text { Re1at1ve Sunspot } \\
\text { Numbers } \\
R_{Z}\end{array}$ & $\begin{array}{c}\text { Dally Values Solar } \\
\text { Flux at } 2800 \mathrm{Mc} \\
\text { Ottawa, Canada } \\
\text { Flux }\end{array}$ \\
\hline 1 & 76 & 124 \\
\hline 2 & 80 & 129 \\
\hline 3 & 69 & 130 \\
\hline 4 & 58 & 131 \\
\hline 5 & 73 & 144 \\
\hline 6 & 90 & 148 \\
\hline 7 & 116 & 157 \\
\hline 8 & 125 & 168 \\
\hline 9 & 125 & 175 \\
\hline 10 & 125 & 200 \\
\hline 11 & 134 & 188 \\
\hline 12 & 132 & 168 \\
\hline 13 & 128 & 180 \\
\hline 14 & 132 & 192 \\
\hline 15 & 133 & 183 \\
\hline 16 & 121 & 174 \\
\hline 17 & 103 & 164 \\
\hline 18 & 82 & 153 \\
\hline 19 & 74 & 150 \\
\hline 20 & 82 & 147 \\
\hline 21 & 65 & 139 \\
\hline 22 & 57 & 127 \\
\hline 23 & 57 & 116 \\
\hline 24 & 41 & 113 \\
\hline 25 & 42 & 111 \\
\hline 26 & 60 & 117 \\
\hline 27 & 58 & 119 \\
\hline 28 & 56 & 117 \\
\hline 29 & 53 & 119 \\
\hline 30 & 63 & 131 \\
\hline Mean: & 87.0 & 147.1 \\
\hline
\end{tabular}

COMERCE - STAMOARDS - BOULDER 


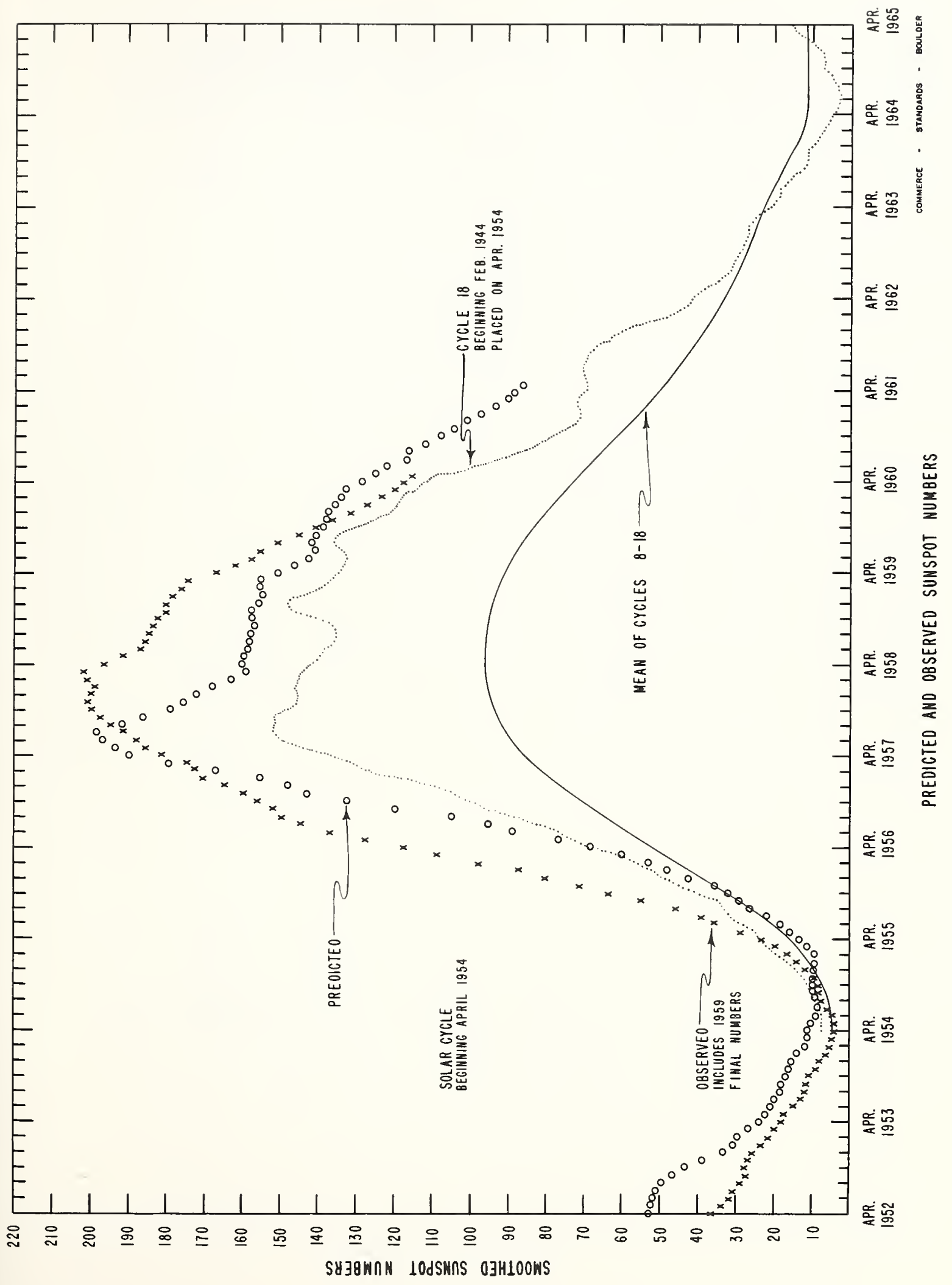




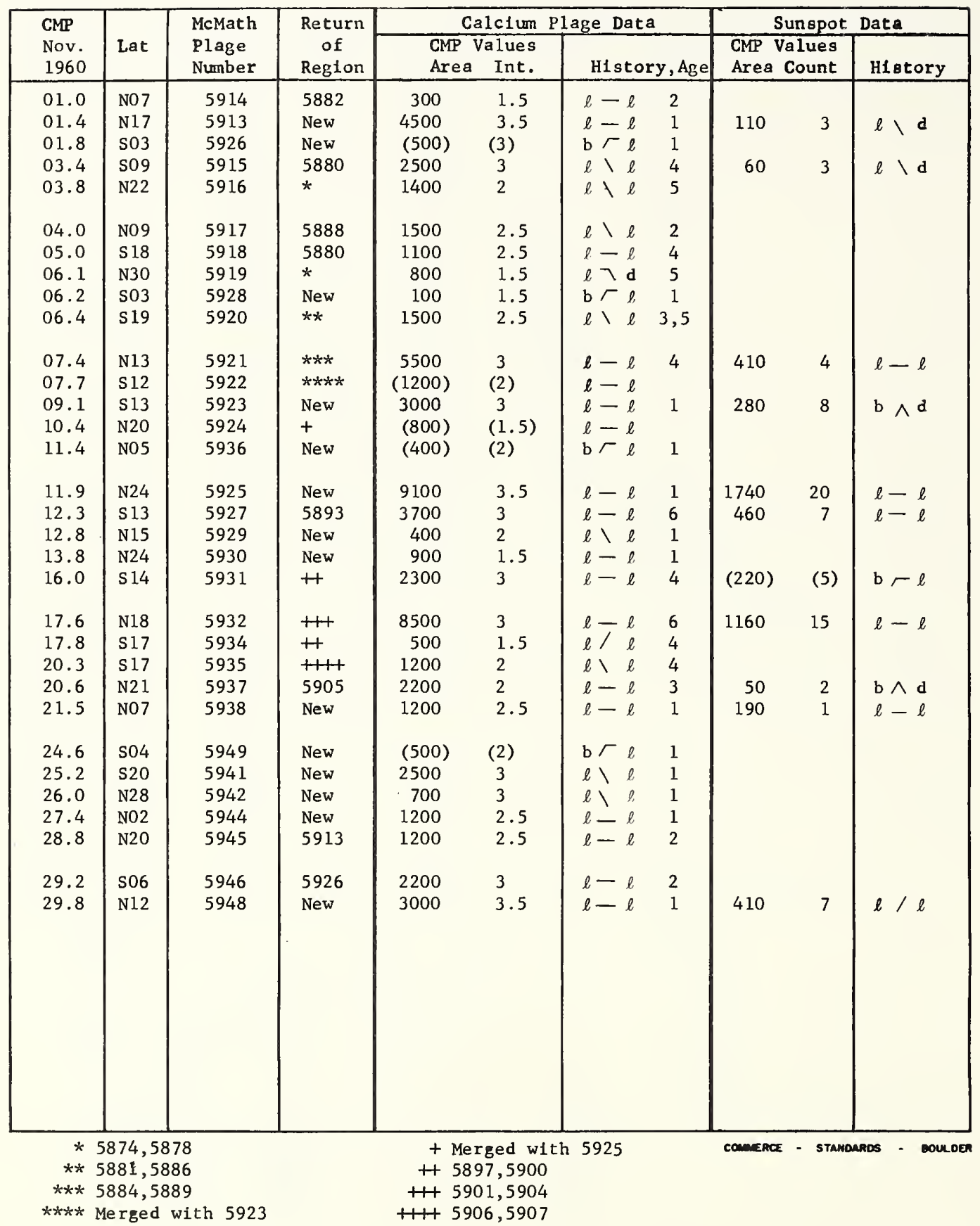




\begin{tabular}{|c|c|c|c|c|c|c|c|}
\hline & $x^{-1}$ & 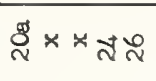 & 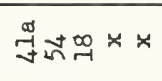 & $\forall \not{x} \vec{m}$ & $m \times \infty \stackrel{m}{m} \underset{\sim}{n}$ & 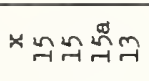 & $\infty \times * \times{ }_{\infty} \times$ \\
\hline 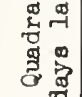 & $x^{\infty}$ & 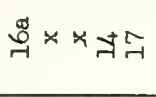 & $\stackrel{\varpi}{\sim} g_{N} \sigma x$ & 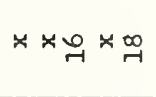 & $\underset{\sim}{ } \not \omega \widehat{N}^{\infty}$ & ×テ્ન & $6 \times x \times g_{-1} x$ \\
\hline 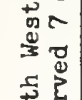 & $5^{-1}$ & 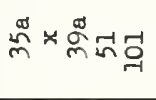 & ร็กูณ* & $\times \underset{్}{\sim} ళ \times \infty$ & 안유유 & × & $q^{\prime}$ \\
\hline 总 & & $\stackrel{\infty}{N} \times \stackrel{\infty}{n} \neq \sigma$ & エ็ & & & $\times 05$ & \\
\hline
\end{tabular}

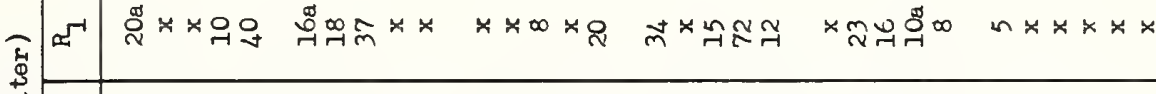

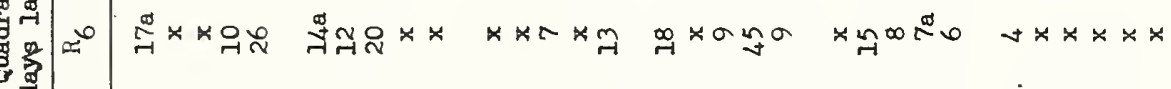
范 兽

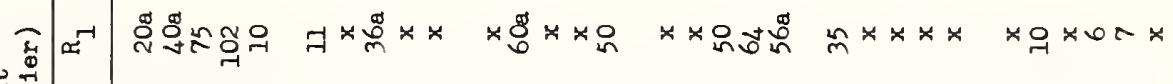
苟

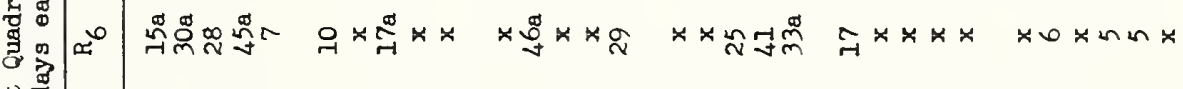
范

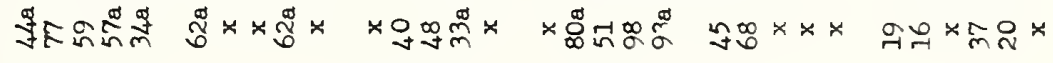

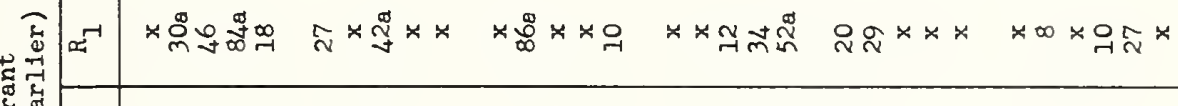

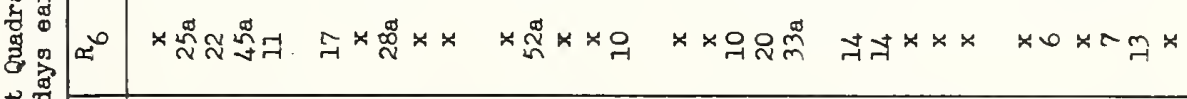

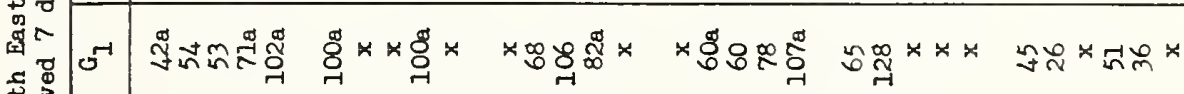
:

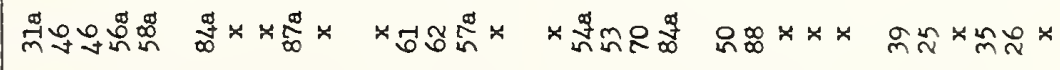

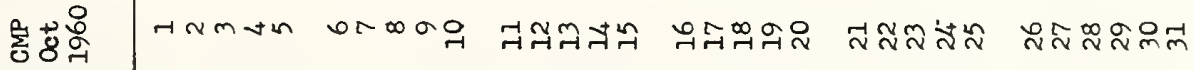


IIc

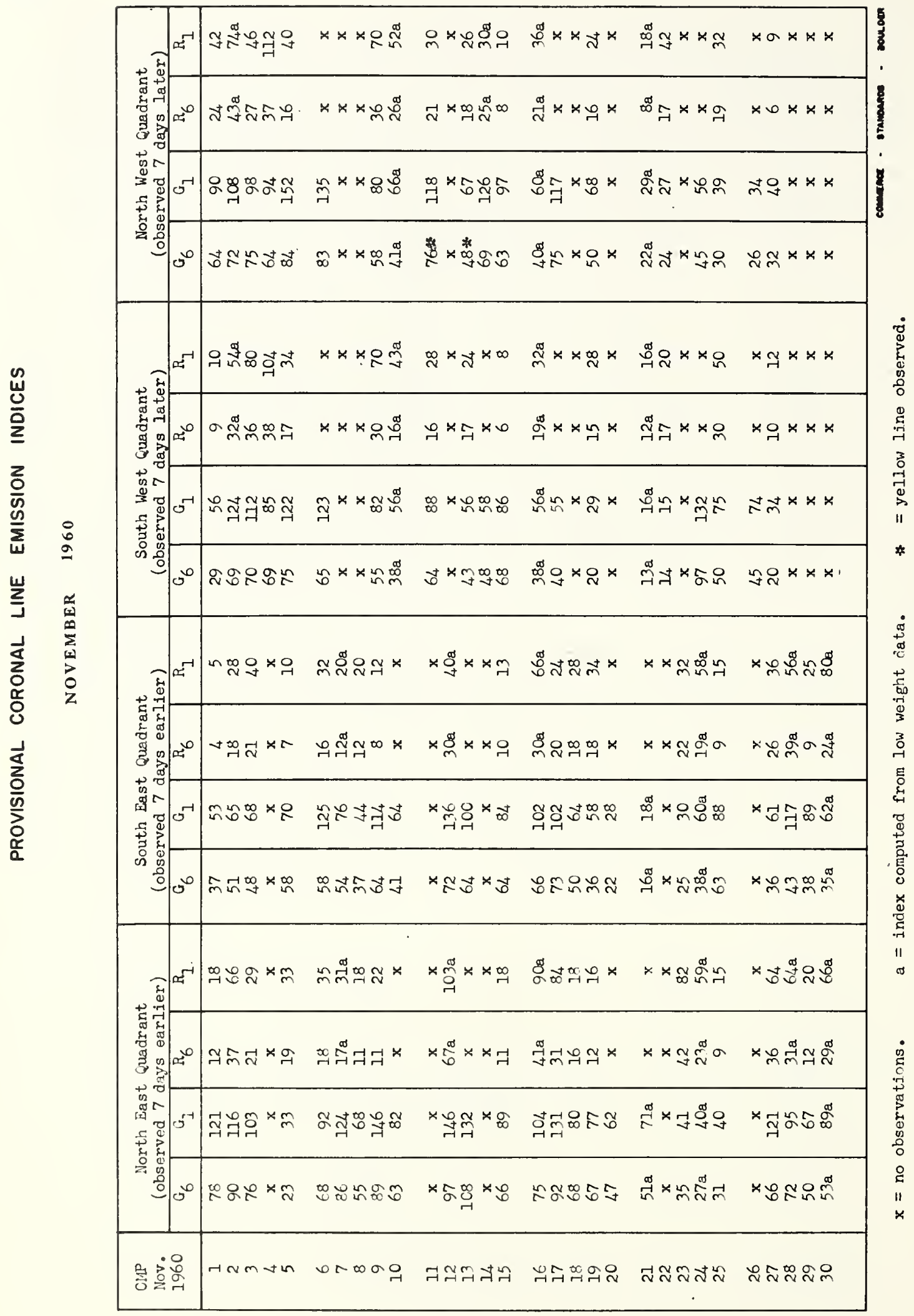




\begin{tabular}{|c|c|c|c|c|c|c|}
\hline 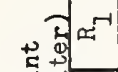 & 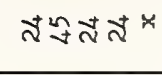 & ำลำ & 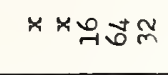 & ヘํㅇำ年 & 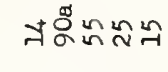 & $\times \infty$ \\
\hline 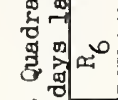 & 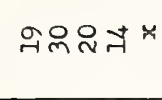 & $\left.\eta^{\infty} \sigma 0\right]^{\infty}$ & メメテษ & 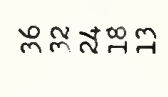 & 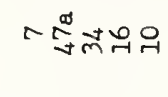 & 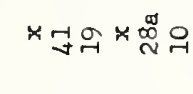 \\
\hline 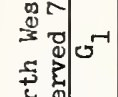 & 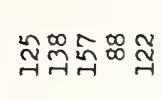 & 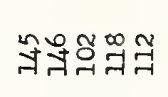 & 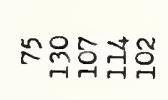 & ర્ન & 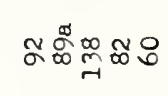 & 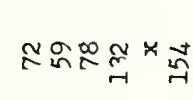 \\
\hline 3 & 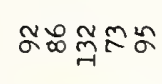 & 엄엉ำ & 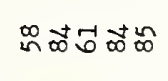 & 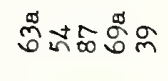 & 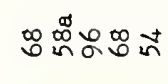 & 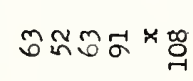 \\
\hline & 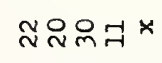 & $\underset{\sim}{2} 001 \stackrel{\infty}{N}$ & トメ文ネন & 축조소ำ & 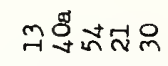 & 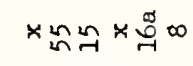 \\
\hline " & 픅어 $\sigma x$ & $\stackrel{\theta}{\sim} \sim \sim 1 \stackrel{\sim}{\sim}$ & $x \times$ न्न & ิ & ๑ & 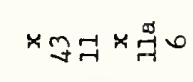 \\
\hline 开 & 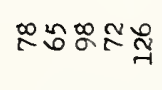 & 号号7788 & 웕워ำ & 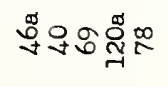 & 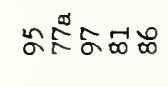 & 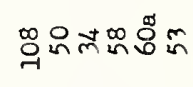 \\
\hline ஸे & ㅅำํํํํำ & 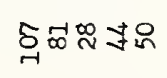 & 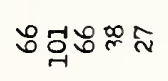 & 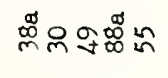 & 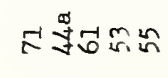 & ๒ m \\
\hline 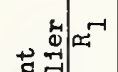 & 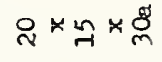 & 공유 * & $\delta \times \delta \times q$ & 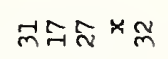 & 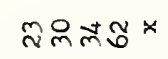 & 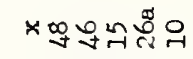 \\
\hline 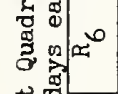 & 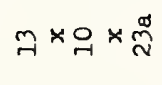 & 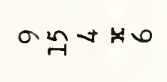 & $\underset{\sim}{s} \times m x+$ & $\hat{\sim}^{0} \pm * 0$ & 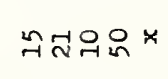 & 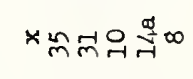 \\
\hline 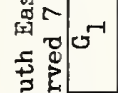 & 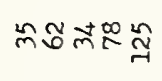 & 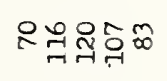 & 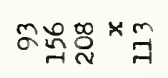 & 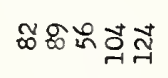 & 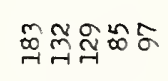 & 국웍 중ㅎㅀ \\
\hline " & 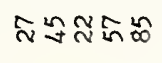 & $\stackrel{\infty}{+} \div \underset{\infty}{\infty}$ & $\Re \infty \% \times$ & 조융유 & 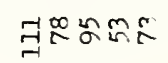 & 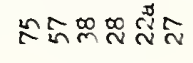 \\
\hline क्⿹勹巳寸 & $\stackrel{N}{*} \times m \times g$ & ఇ유묵유 & 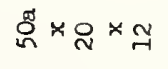 & $8 \exists \leftarrow \times$ & 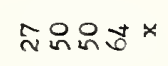 & $\times 8 \% \times$ \\
\hline 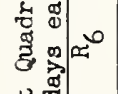 & 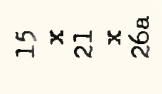 & $\exists \operatorname{man} \sigma x$ & $\underset{\sim}{\simeq} \times \sim \times \infty$ & 今+ & 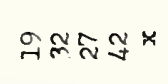 & 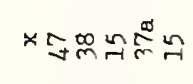 \\
\hline 疍 & 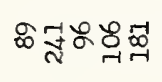 & 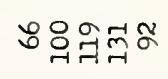 & 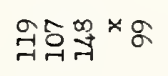 & 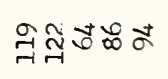 & 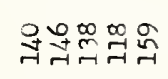 & 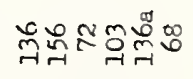 \\
\hline "0 & S쁌ำ & 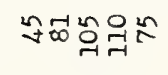 & $\infty_{\infty}^{\infty} \infty_{\infty}^{\infty} \infty \times \pi$ & 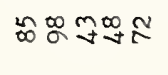 & 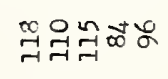 & ๓ ホテ็ \\
\hline 혼 곡융 & Hom ton & 시유 & 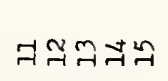 & 눅용요 & 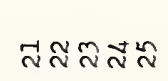 & ํํำ \\
\hline
\end{tabular}


II

\begin{tabular}{|c|c|c|c|c|c|c|}
\hline + की की & $\stackrel{\infty}{\sim} \stackrel{\infty}{\sim} \times \infty_{\infty}^{\infty} \sim$ & $\times \underset{\sim}{\infty} \times \times \underset{\sim}{\stackrel{f}{\widetilde{I}}}$ & $\times \underset{\forall}{*} \times \underset{\sim}{*}$ & $\times \mathbb{\sim} \underset{N}{ } \times O_{\infty}$ & 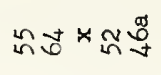 & సాఖ్య \\
\hline 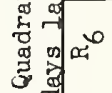 & 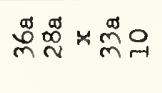 & 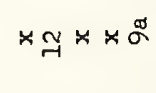 & x억어 x 귁 & 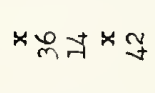 & $\widehat{\sim} m^{+} \approx \widetilde{\sim}$ & ส \\
\hline 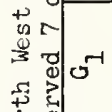 & న. న్రం & नี & : & × & 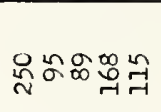 & 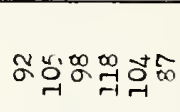 \\
\hline
\end{tabular}

包

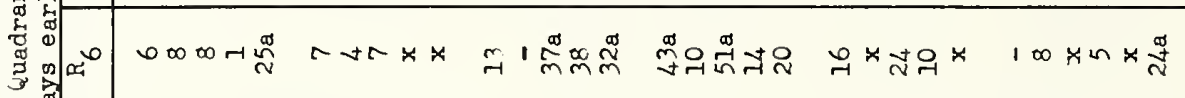

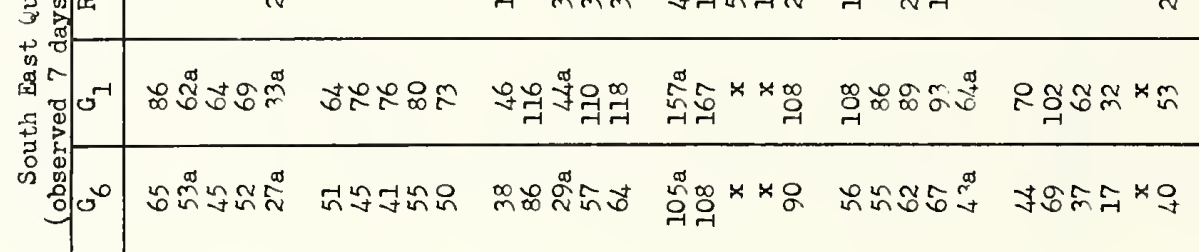

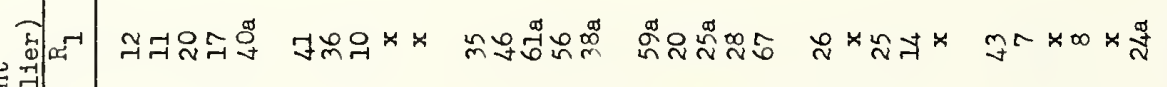

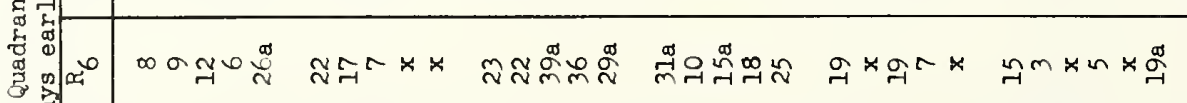

\begin{tabular}{|c|c|c|c|c|c|c|}
\hline 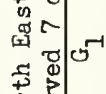 & 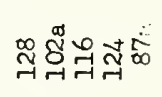 & 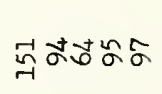 & 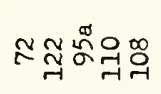 & 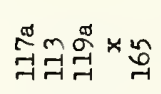 & 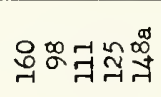 & 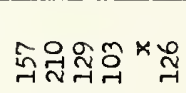 \\
\hline 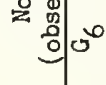 & 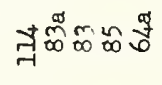 & 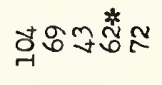 & 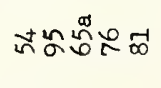 & 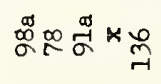 & 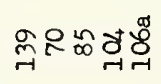 & 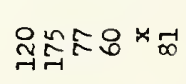 \\
\hline 롱 룽유 & HNM th & orwa요 & 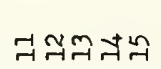 & 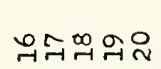 & సָָָస̃ & 오요 \\
\hline
\end{tabular}




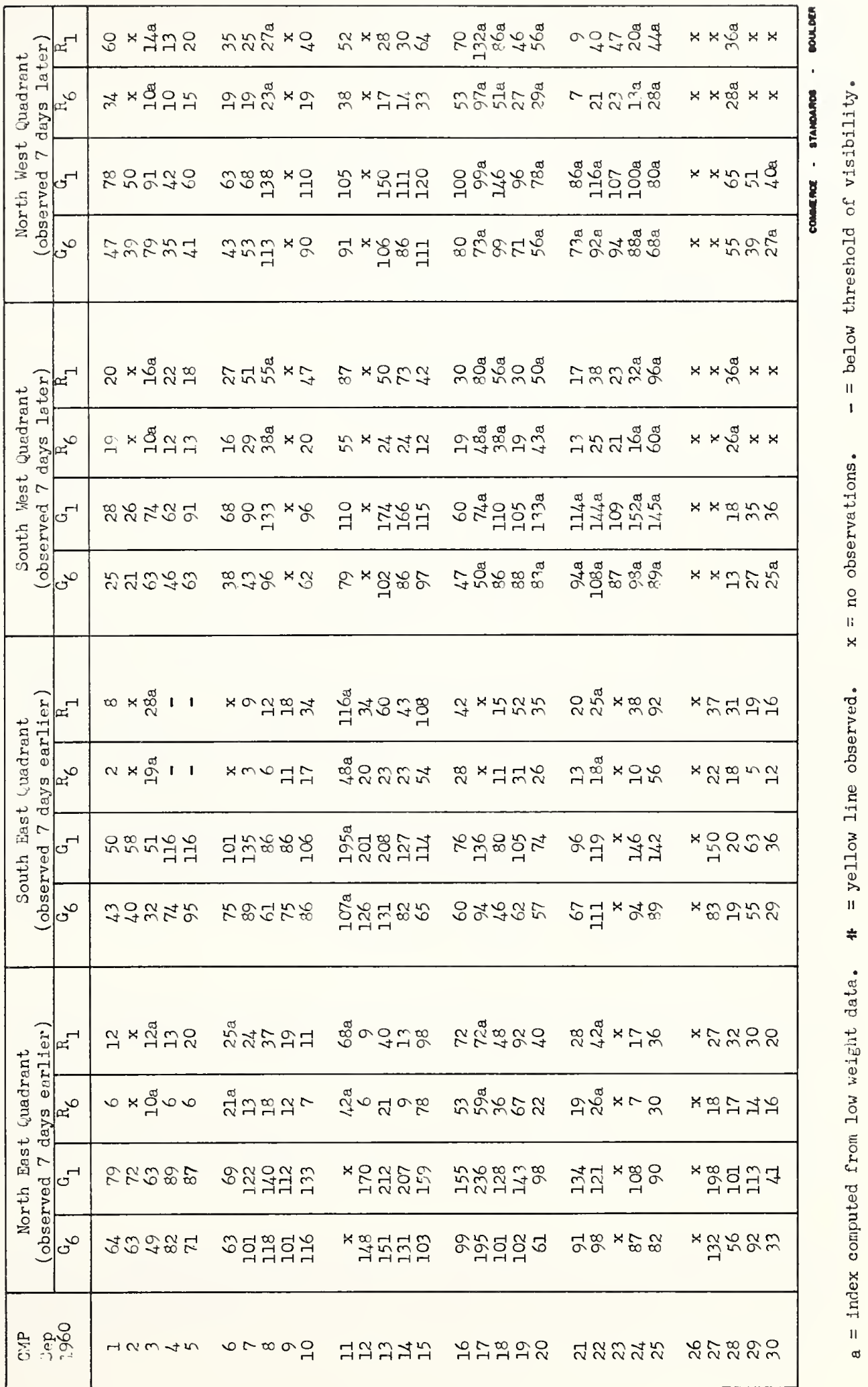




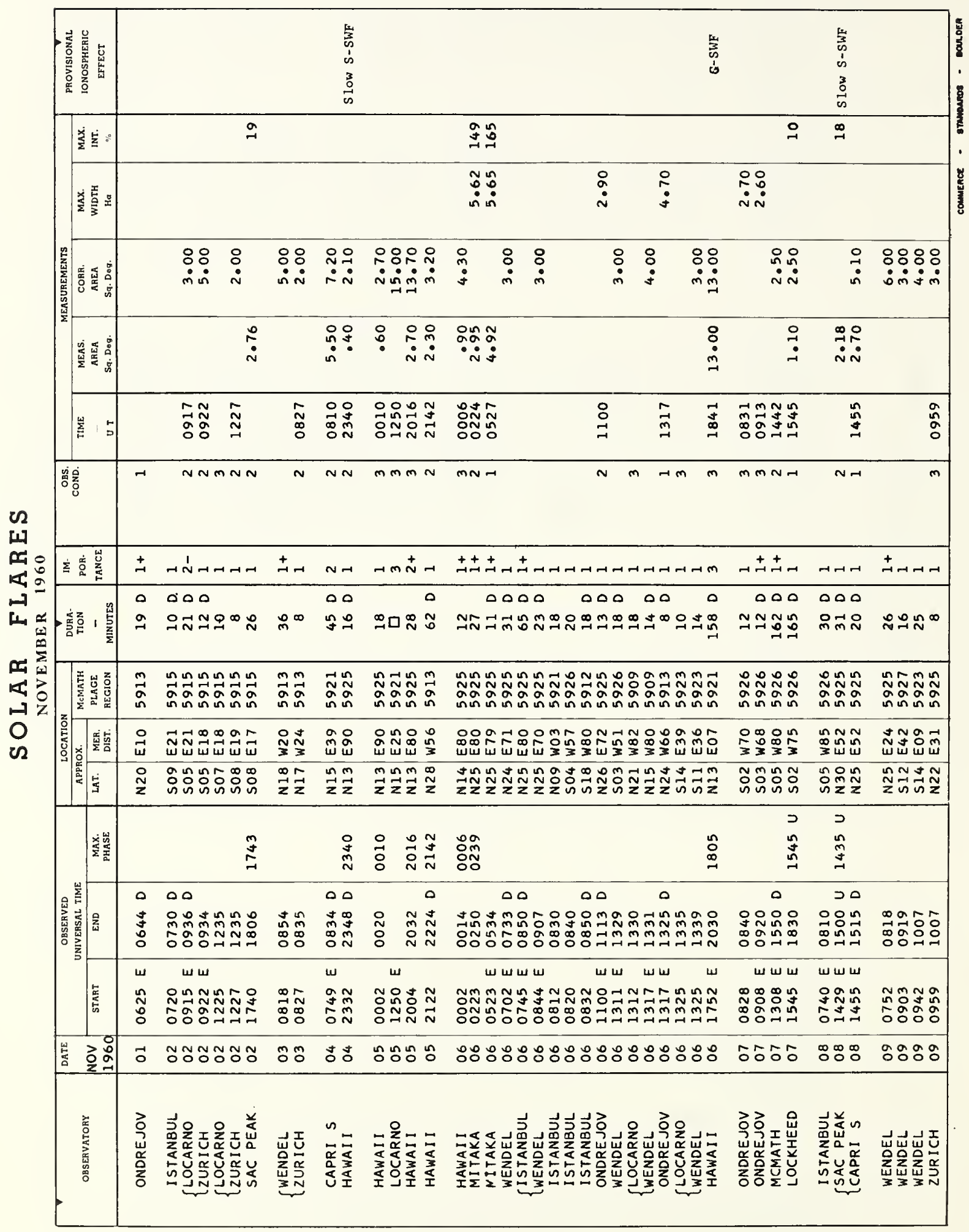




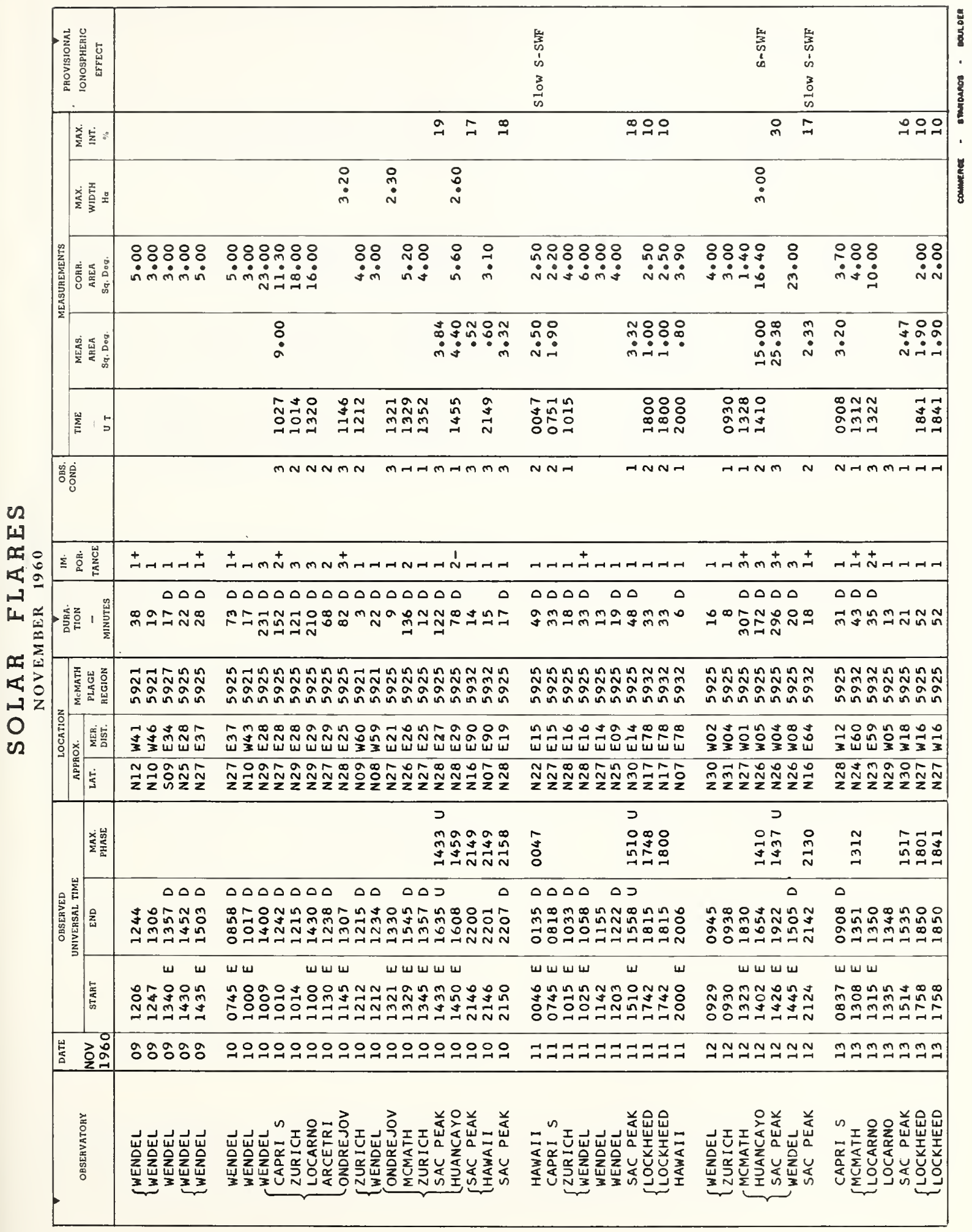




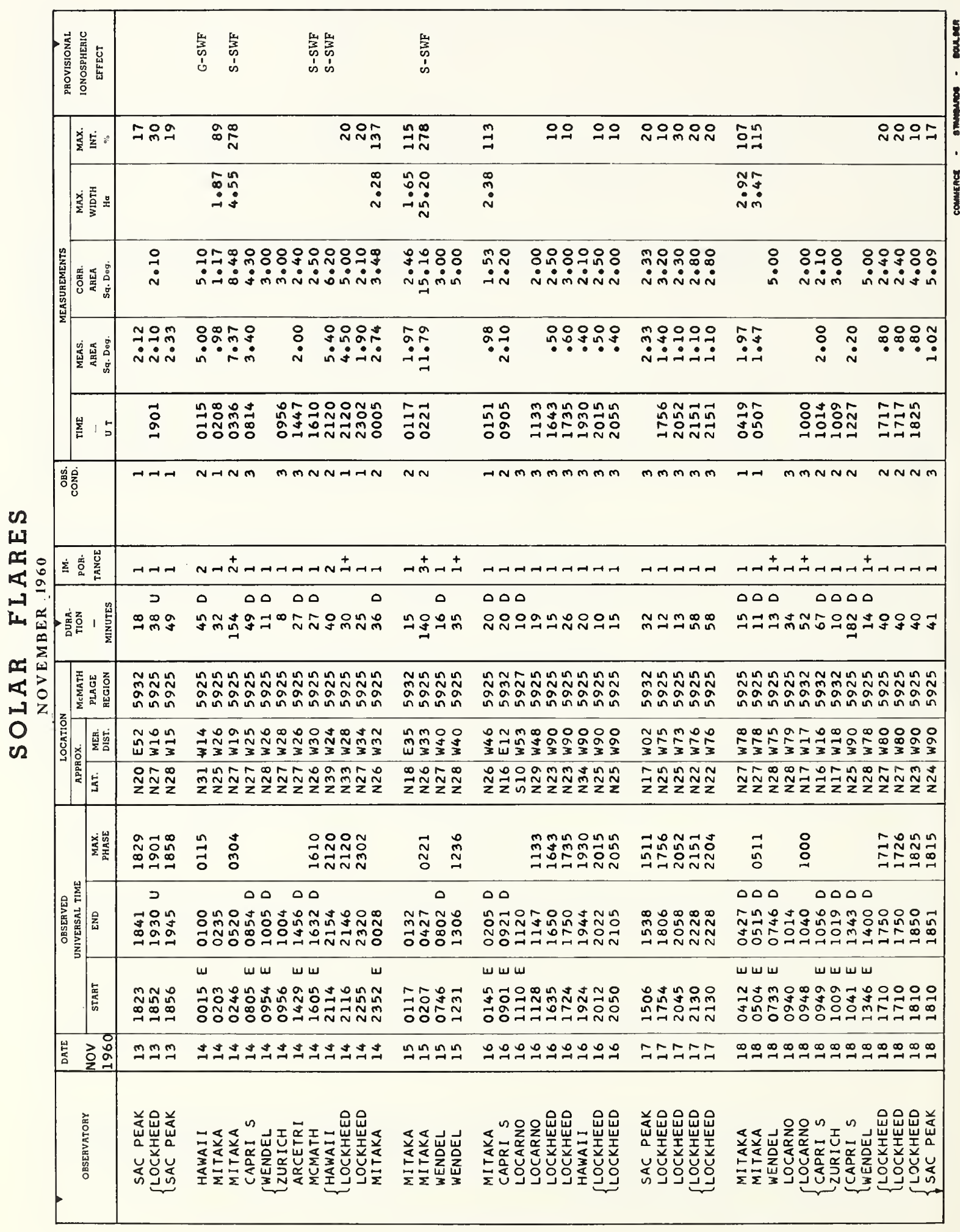




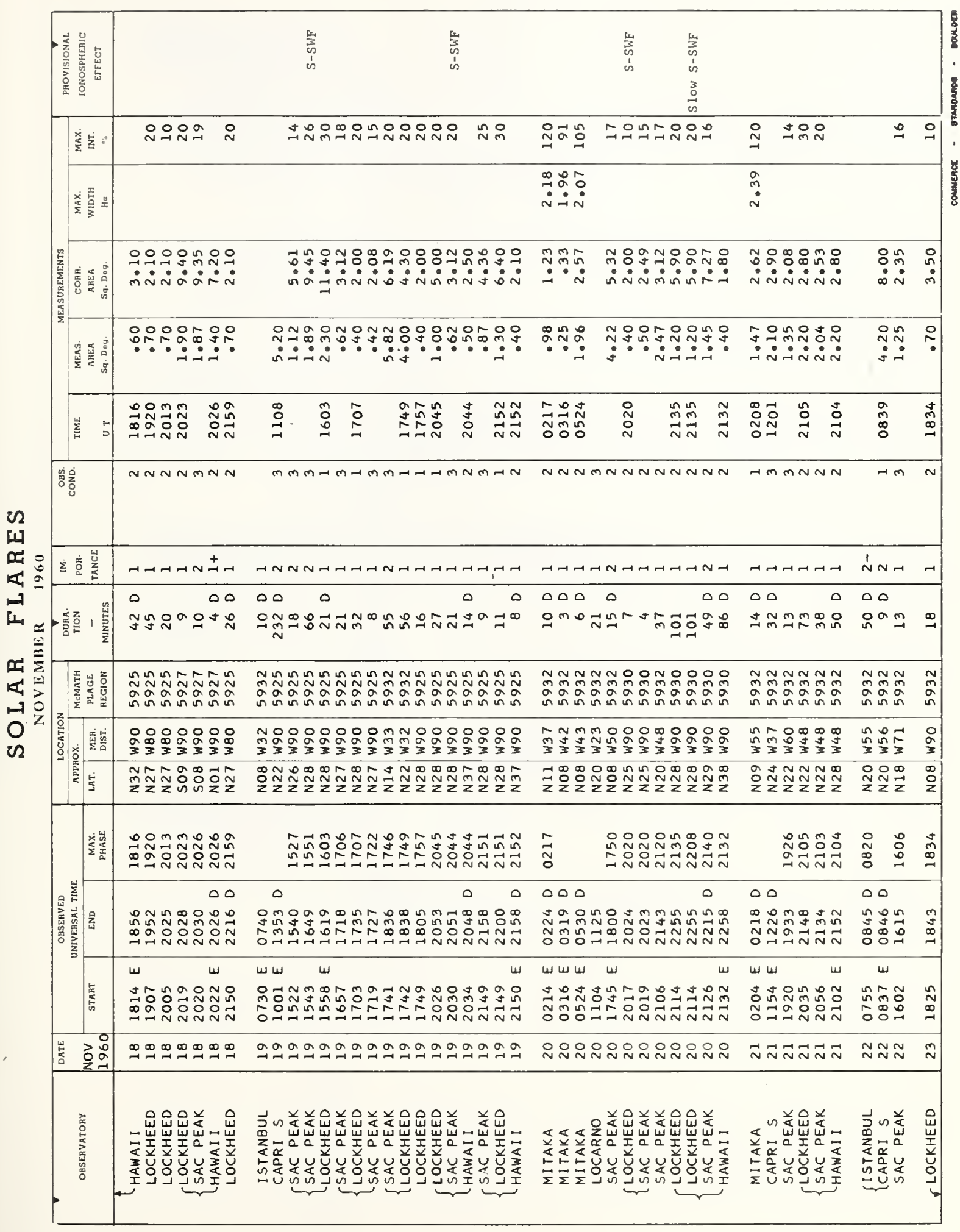




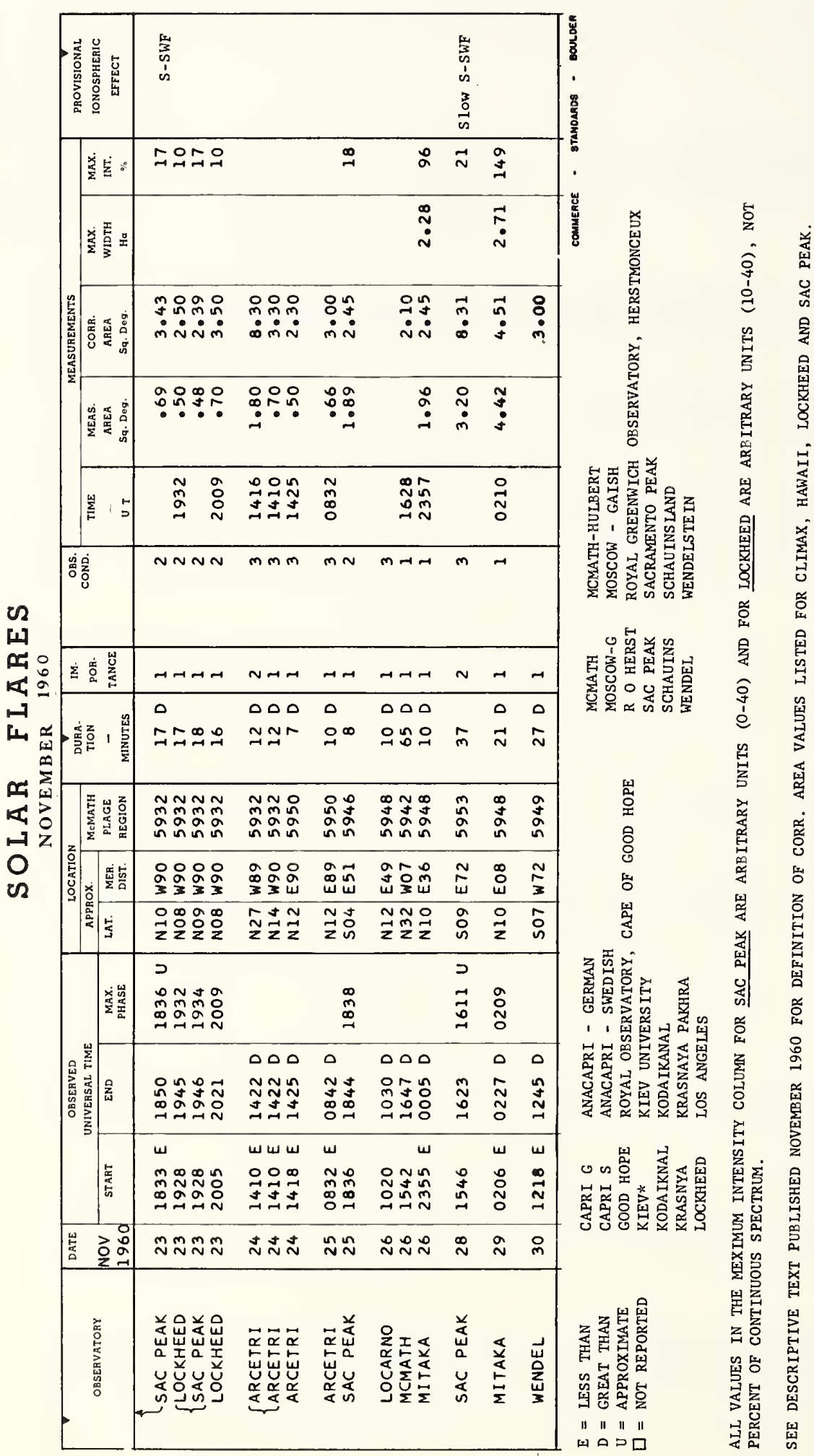




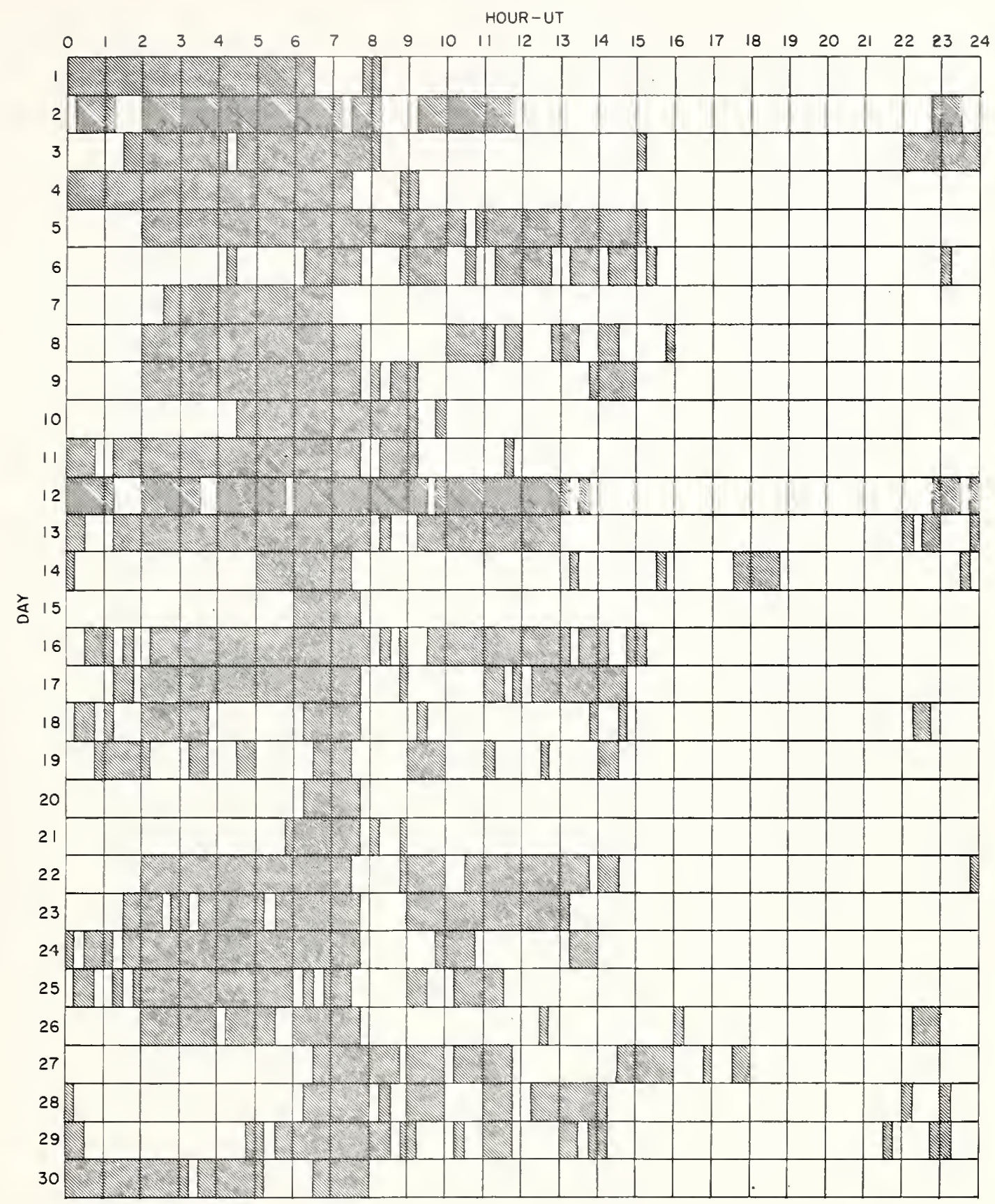

Stations Include:

Anacapri (Swedish)

Arcetri

Hawai

Huancayo
Istanbu1 Kodalkanal Lockheed McMath
Mitaka

Ondrejov

Royal Greenwich Observatory

Herstmonceux

Sacramento Peak 


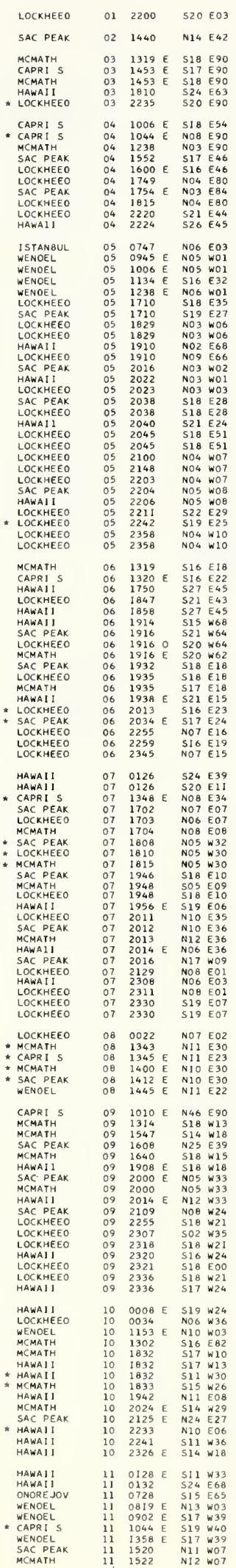

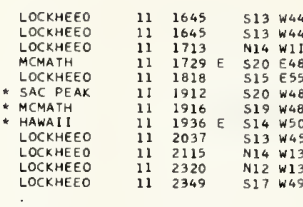

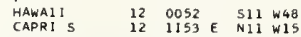

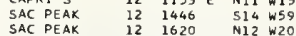

LOCXNEEO 12 1625 E $N 13$ W19

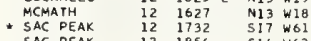

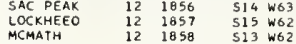

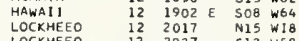

SAC PEAK $\quad 12 \quad 2158 \quad 513$ E40

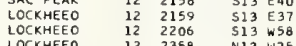

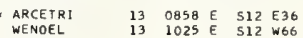

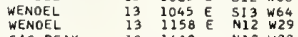

SAC PEAK
MCAATH
MCA

WENOEL
SAC PEAK

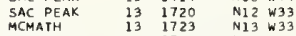

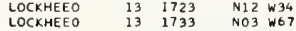

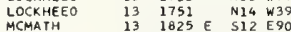

MCAAH 13 1937 E 112 E90

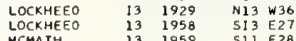

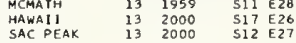

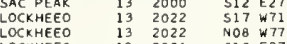

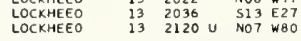

- WEEOEL 140951 E 513 E17

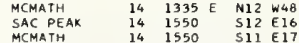

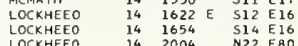

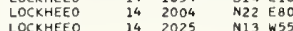

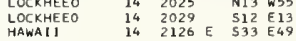

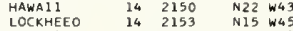

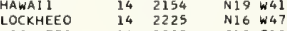

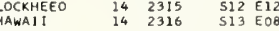

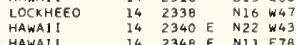

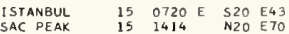

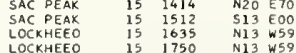

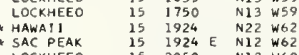

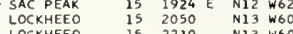

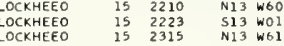

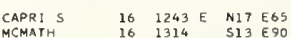

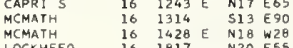

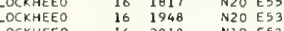

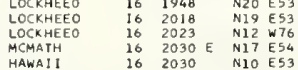

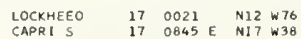

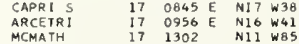

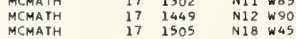

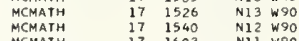

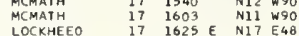

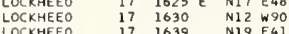

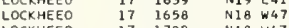

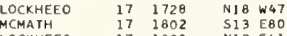

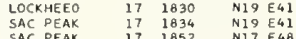

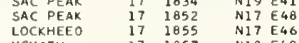

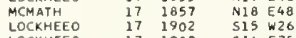

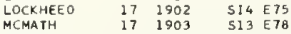

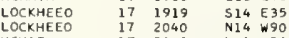

$172042 \quad \mathrm{~N} 11 \mathrm{w90}$

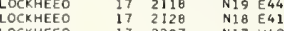

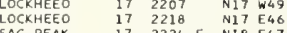

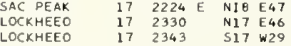

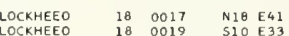

WENOEL

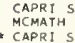

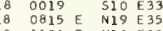

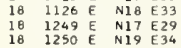

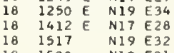

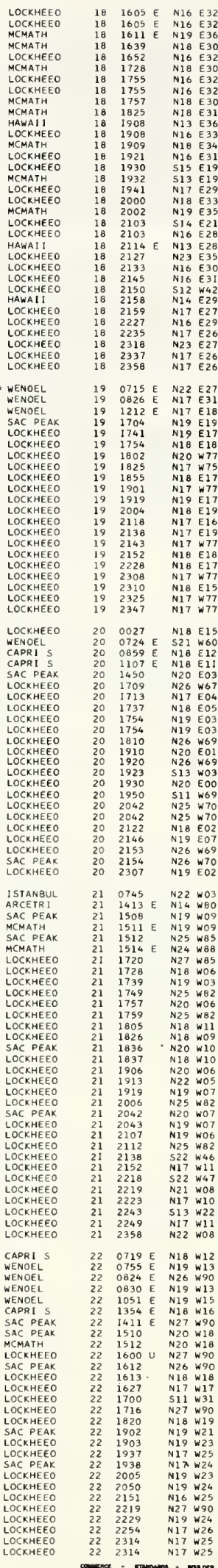


Noted as follows: Date-Universal Time-Coordinates OCTOBER 1960

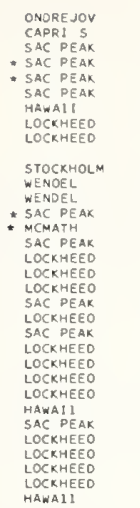

\begin{tabular}{|c|c|c|}
\hline & & \\
\hline & 1234 & \\
\hline & 1404 & $N 20$ \\
\hline & 1512 & N2O \\
\hline & 1544 & N2O \\
\hline & & $\mathrm{N} 20$ \\
\hline & 1751 E & N29 \\
\hline & $\begin{array}{l}2208 \\
2241\end{array}$ & $\begin{array}{l}\text { N18 } \\
\text { N19 }\end{array}$ \\
\hline & $0905 E$ & $N 18$ \\
\hline & 1153 & \\
\hline & 1210 & N21 \\
\hline & $\begin{array}{l}1454 \\
1455\end{array}$ & $\begin{array}{l}\text { N16 } \\
\text { N16 }\end{array}$ \\
\hline & & $\mathrm{N} 20^{\circ}$ \\
\hline & 161 & N22 \\
\hline & 164 & N22 \\
\hline & 171 & N22 \\
\hline & & $N 20$ \\
\hline & $\begin{array}{l}172 \\
172\end{array}$ & N2O \\
\hline & $\begin{array}{l}172 \\
175\end{array}$ & $\begin{array}{l}\text { N2O } \\
\text { N22 }\end{array}$ \\
\hline & 183 & $N_{19}$ \\
\hline & 184 & N22 \\
\hline & $\begin{array}{l}194 \\
190\end{array}$ & N23 \\
\hline & 191 & \\
\hline & & \\
\hline & 19. & \\
\hline & & $\begin{array}{l}\mathrm{N} 19 \\
\mathrm{~N} 21\end{array}$ \\
\hline & $\begin{array}{l}215 \\
222\end{array}$ & $\begin{array}{l}\mathrm{N} 21 \\
\mathrm{~N} 18\end{array}$ \\
\hline & 2227 & N26 \\
\hline
\end{tabular}

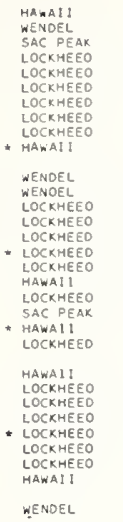

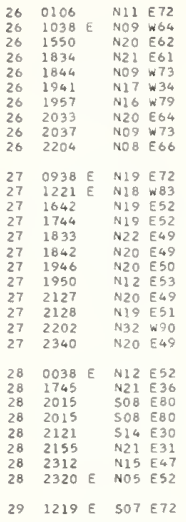

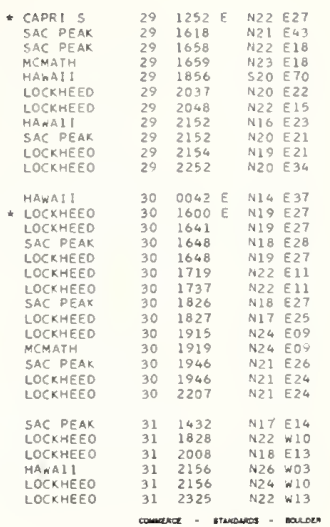

*Rated ao flare of smportance $\geq 1$ by other observatories. (See CRPL-F 195 Part B for November 1960), 


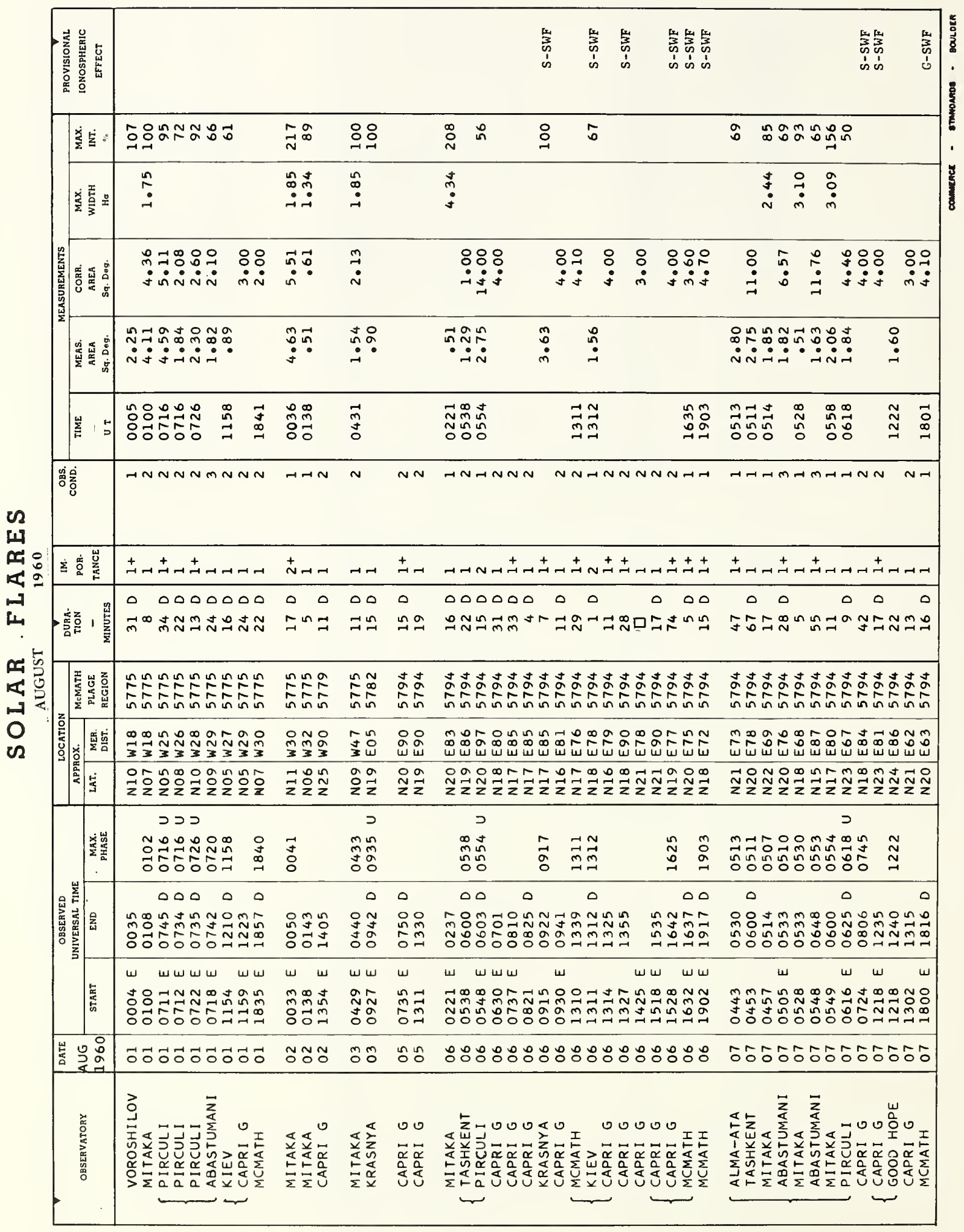




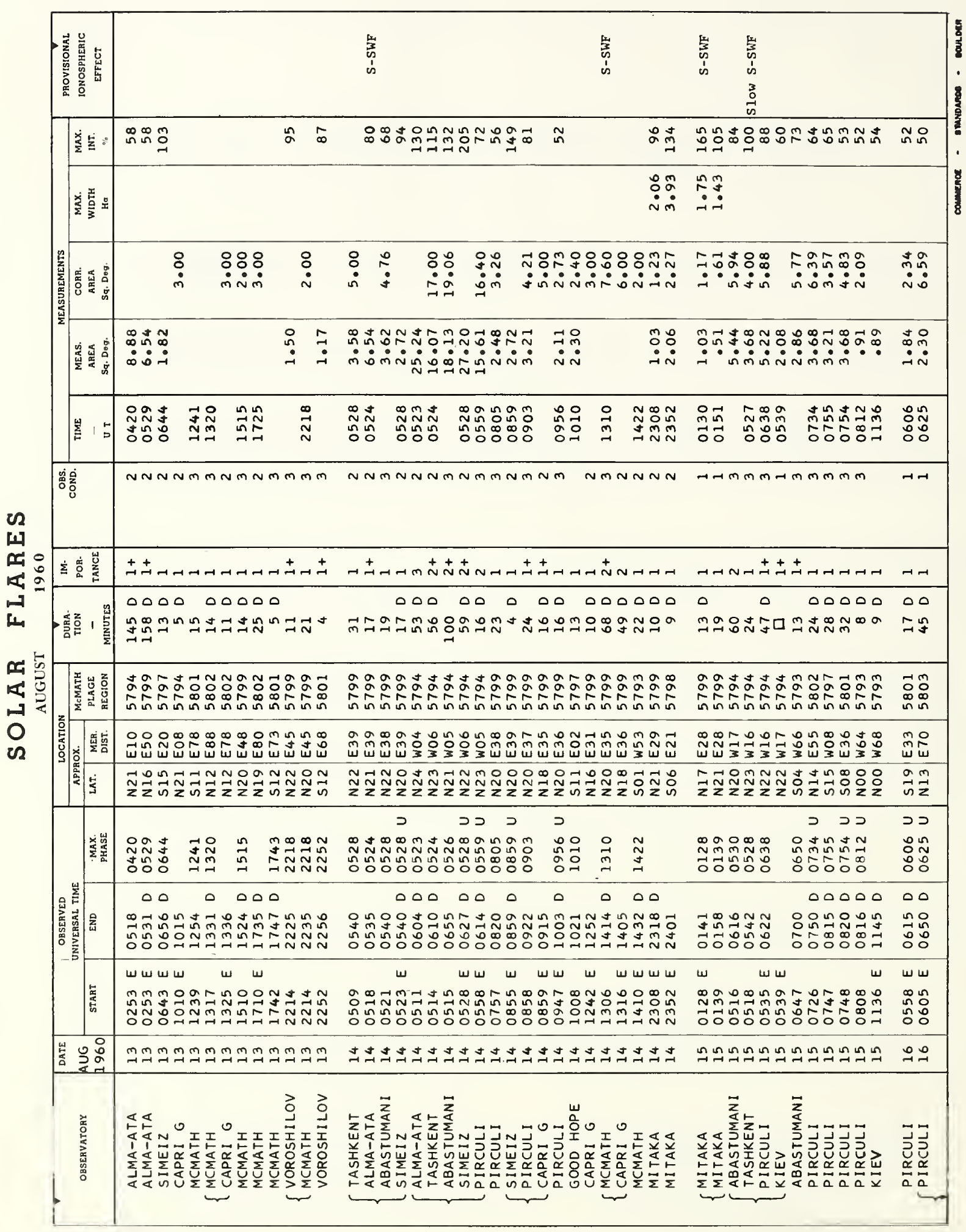




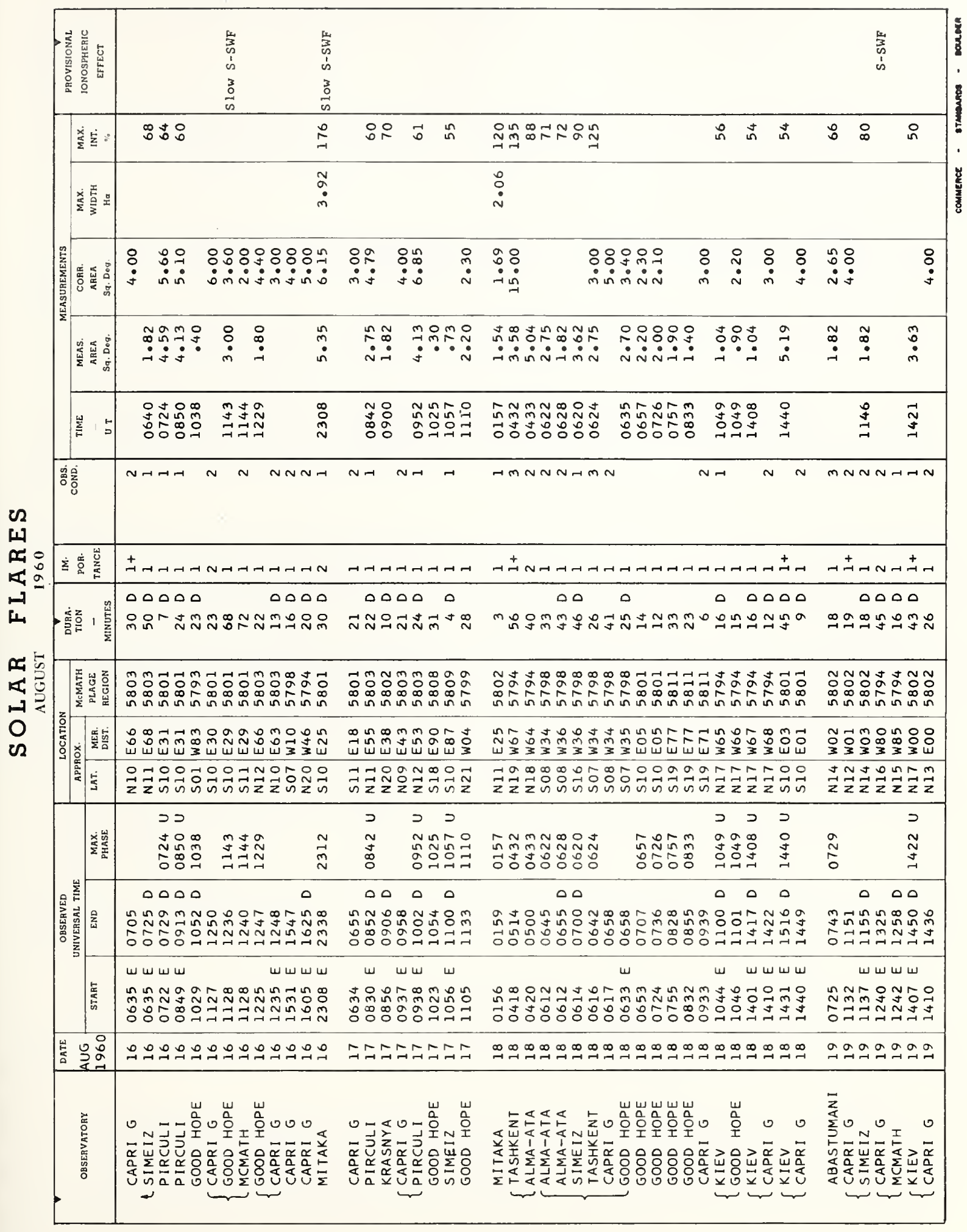




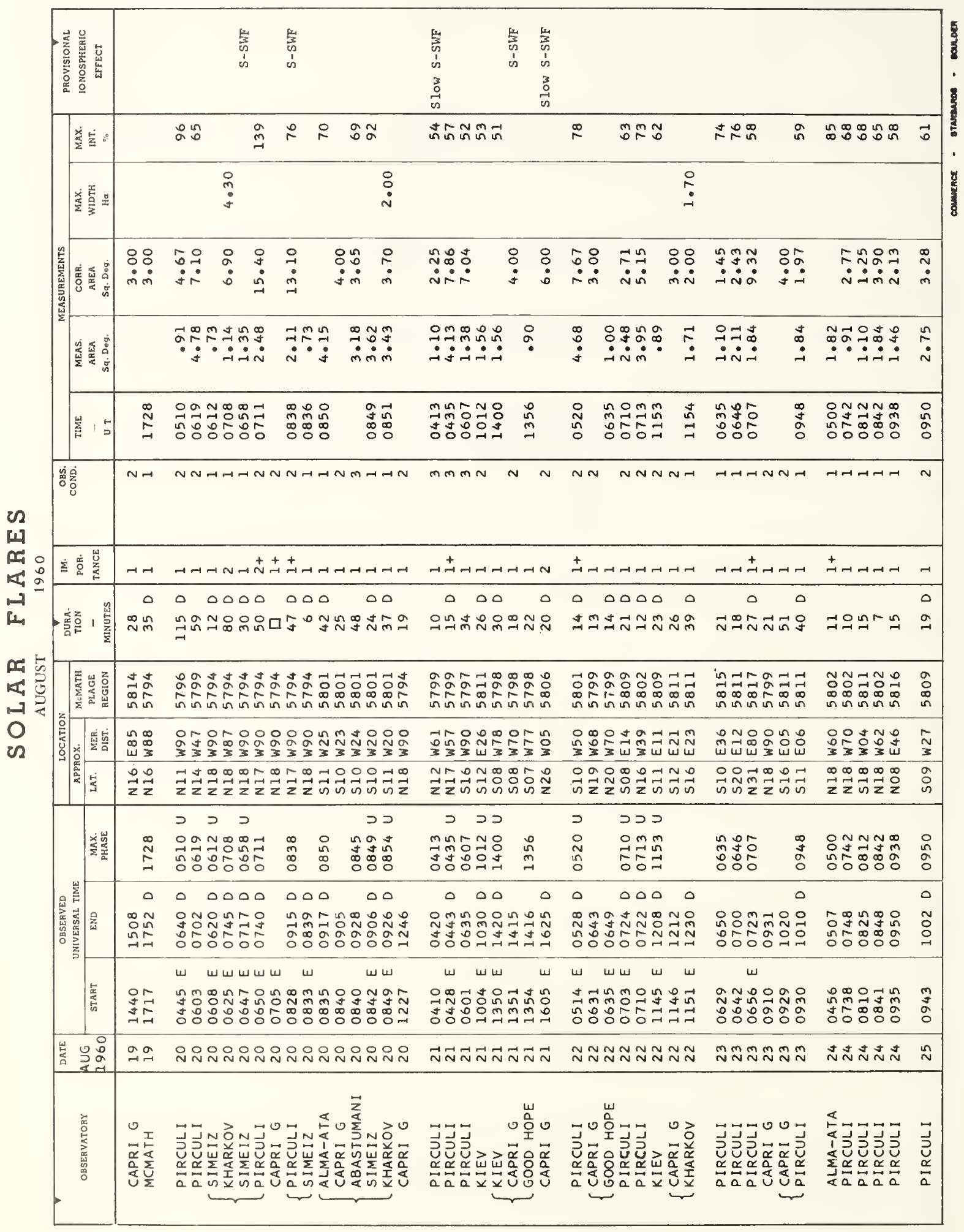




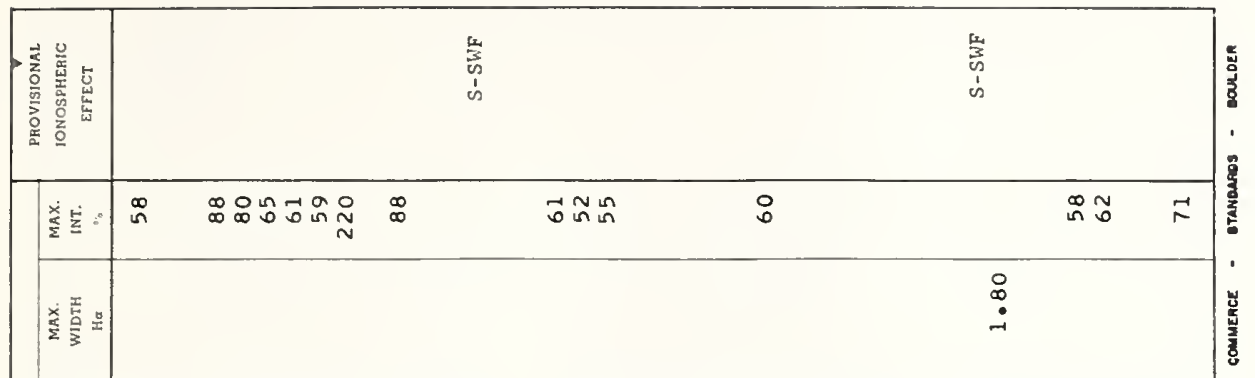

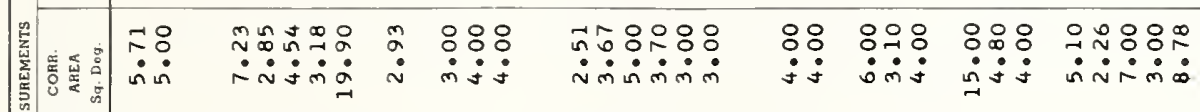
(1)

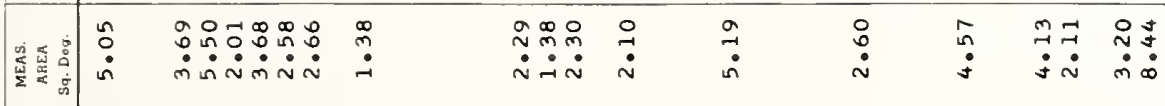

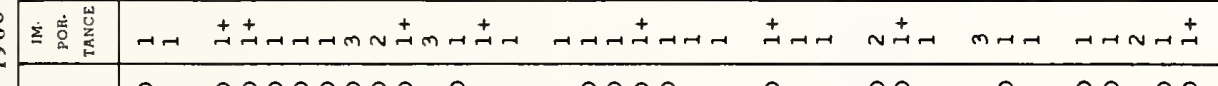
崖等

然尊

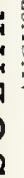

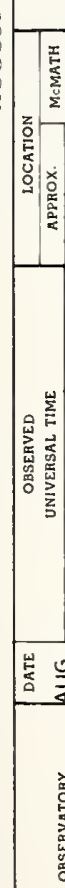

$\stackrel{\substack{5 \\:}}{\infty}$

z

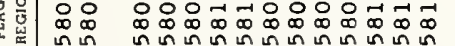

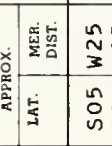

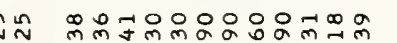

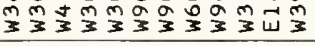

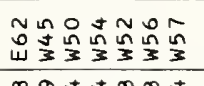

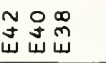

กิกับ

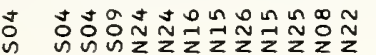

$$
\supset \supset
$$

离嵌

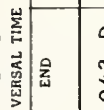

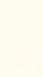

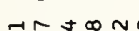

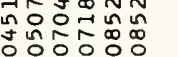

DODODO O

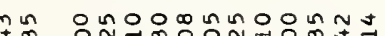

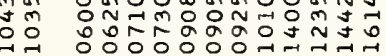

шшшш ш ш

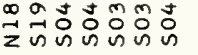

$\supset$

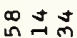

ơ

0

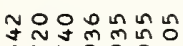

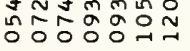

шшш

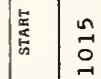

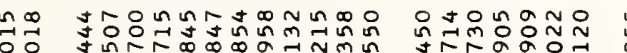

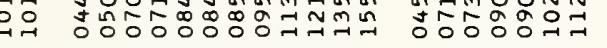

昰式

$\infty$ 웅

这

正药

orin onana

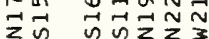

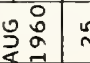

$\stackrel{n}{\sim}$

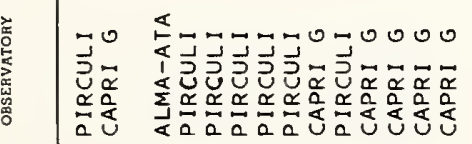

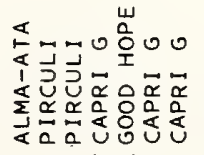

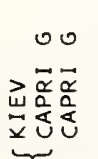

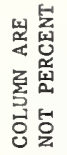

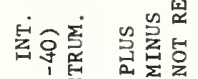

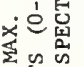

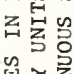

¿ 1 i'

z歪思

受

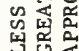

1,1

모

茞

杯

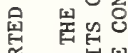

叴穹置

弹究告

葬点

帒密

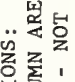

눙워

遥密。

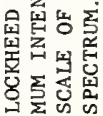


HOUR - UT

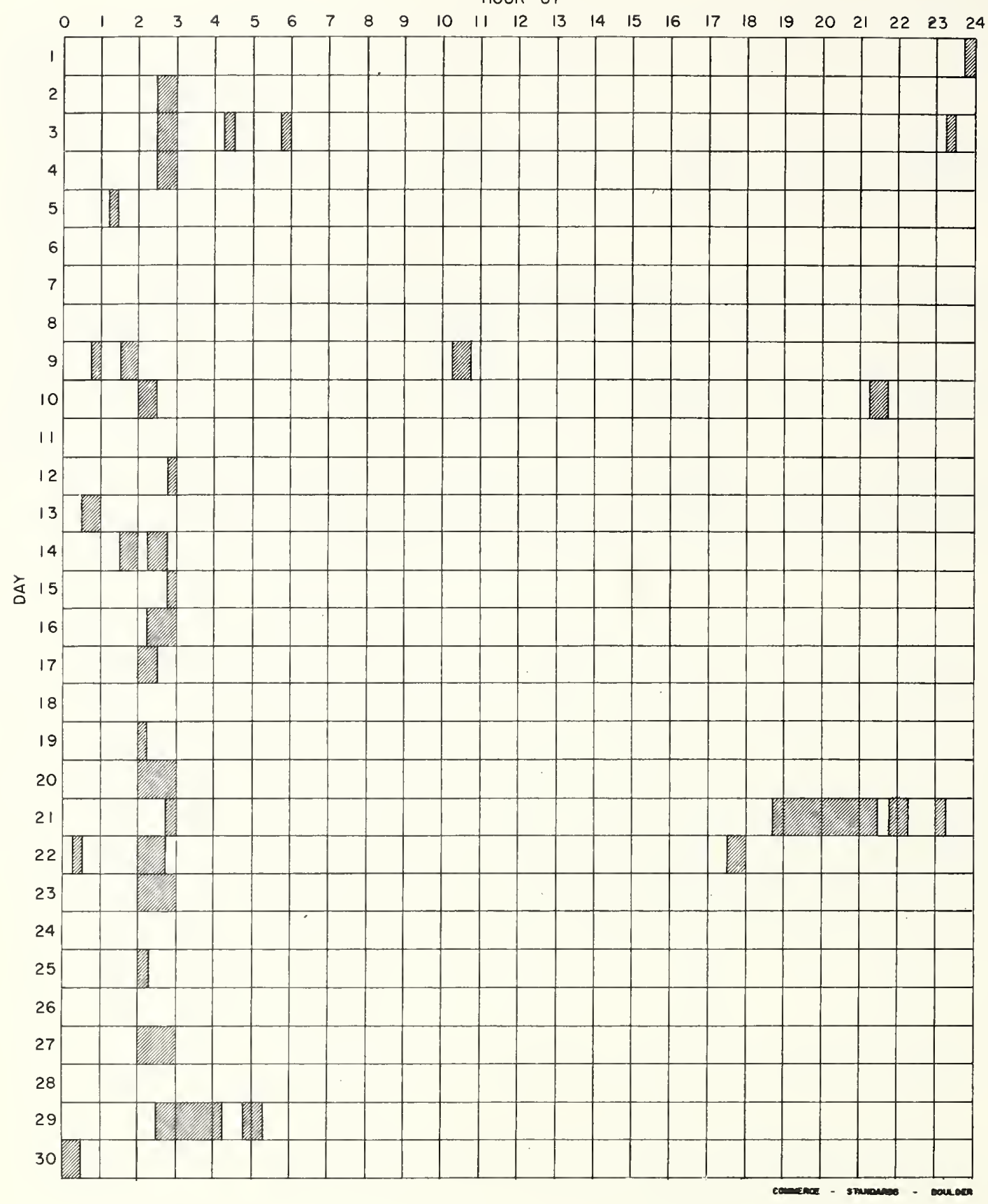

Stations Include:

$\begin{array}{lllll}\text { Abastuman1 } & \text { Hawa11 } & \text { Kodaikanal } & \text { Nlzamiah } & \text { Sacramento Peak } \\ \text { Alma Ata } & \text { Huancayo } & \text { Krasnaya Pakhra } & \text { Ondrejov } & \text { Simeiz } \\ \text { Anacapr1 (Swédish) } & \text { Istanbul } & \text { Lockheed } & \text { Plrcul1 } & \text { Tashkent } \\ \text { Arcetri } & \text { Kharkov } & \text { McMath } & \text { Royal Greenwich Observatory Uccle } \\ \text { Good Hope } & \text { Kiev GAO } & \text { Moscow - G } & \text { Herstmonceux } & \text { Voroshilov }\end{array}$




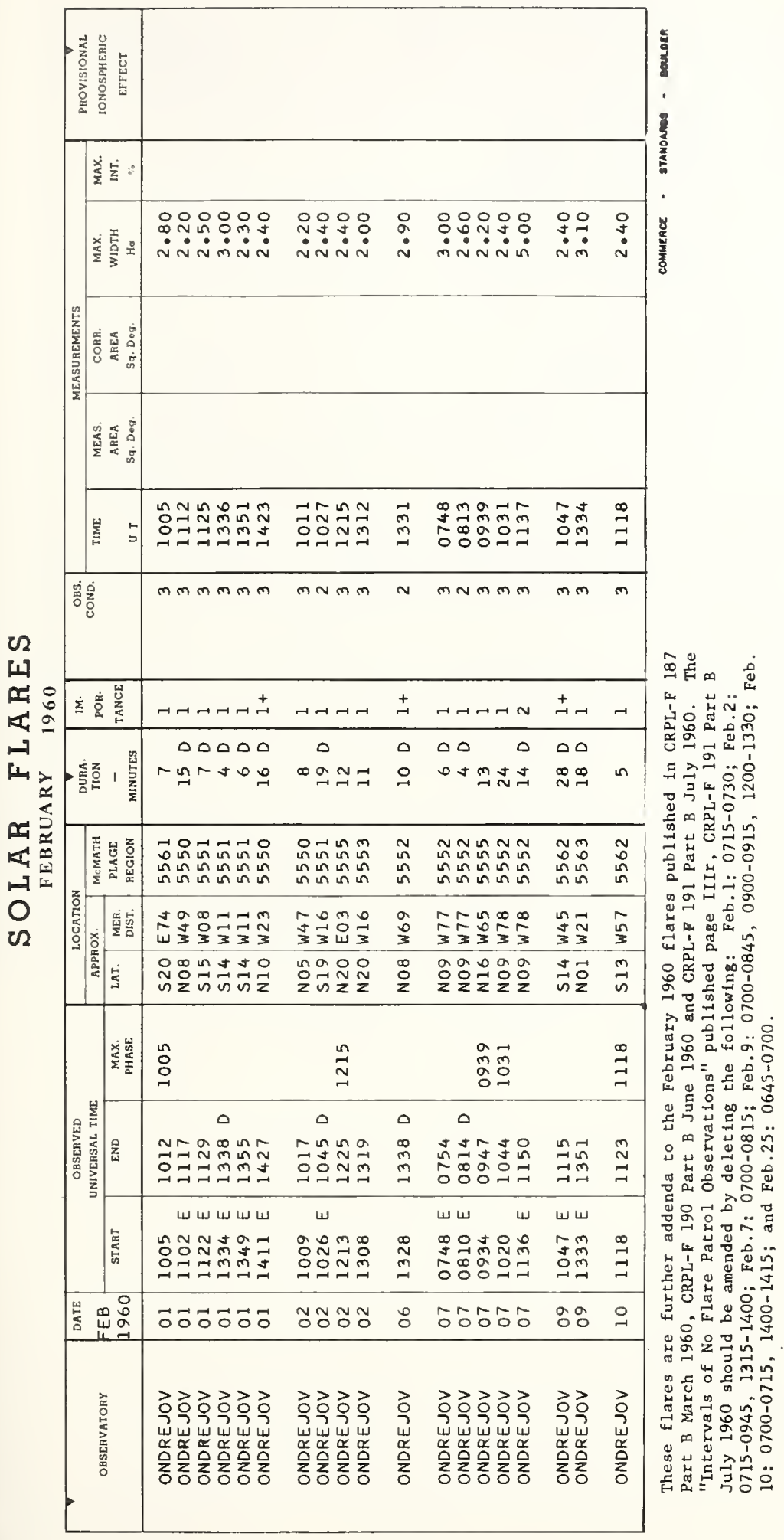


(SHORT-WAVE RADIO FADEOUTS)

OCTOBER 1960

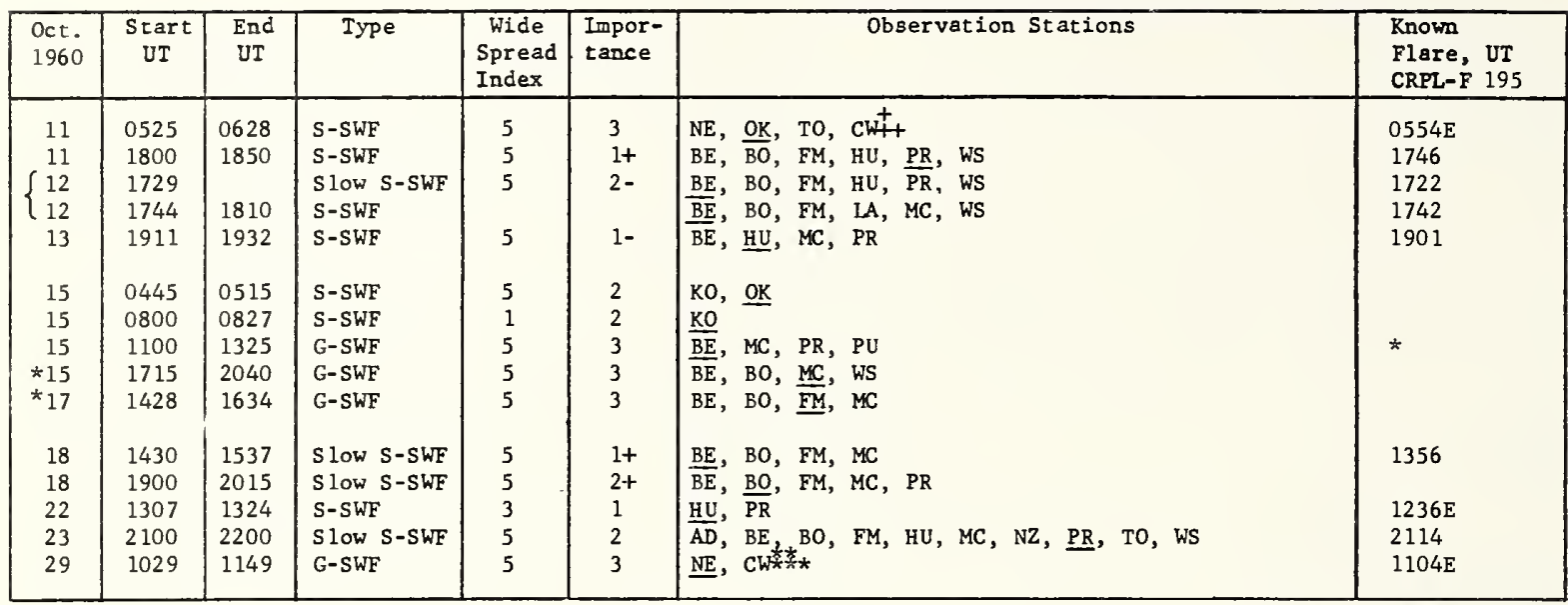

COMnence - STAMDandos - DOULDE

KO = Kodaikana 1 , Ind $1 \mathrm{a}$

$\mathrm{LA}=$ Los Angeles, California

$\mathrm{NZ}=$ New Zealand Post and Telegraph Department

$\mathrm{TO}=$ Hiraiso Radio Wave Observatory, Japan

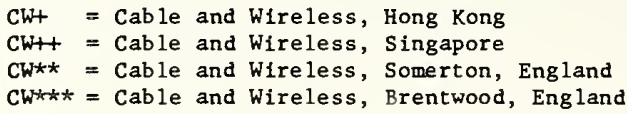

* These events strange, not seen at Puerto Rico, may be due to MUF fallures or changes of mode and not solar flare effects. 
$\left(\begin{array}{l}\text { Sudden Cosmic Noise Absorption } \\ \text { Sudden Enhancements Of Atmospherics } \\ \text { Solar Noise Bursts At } 18 \mathrm{Mc} \text {. }\end{array}\right)$

OCTOBER 1960

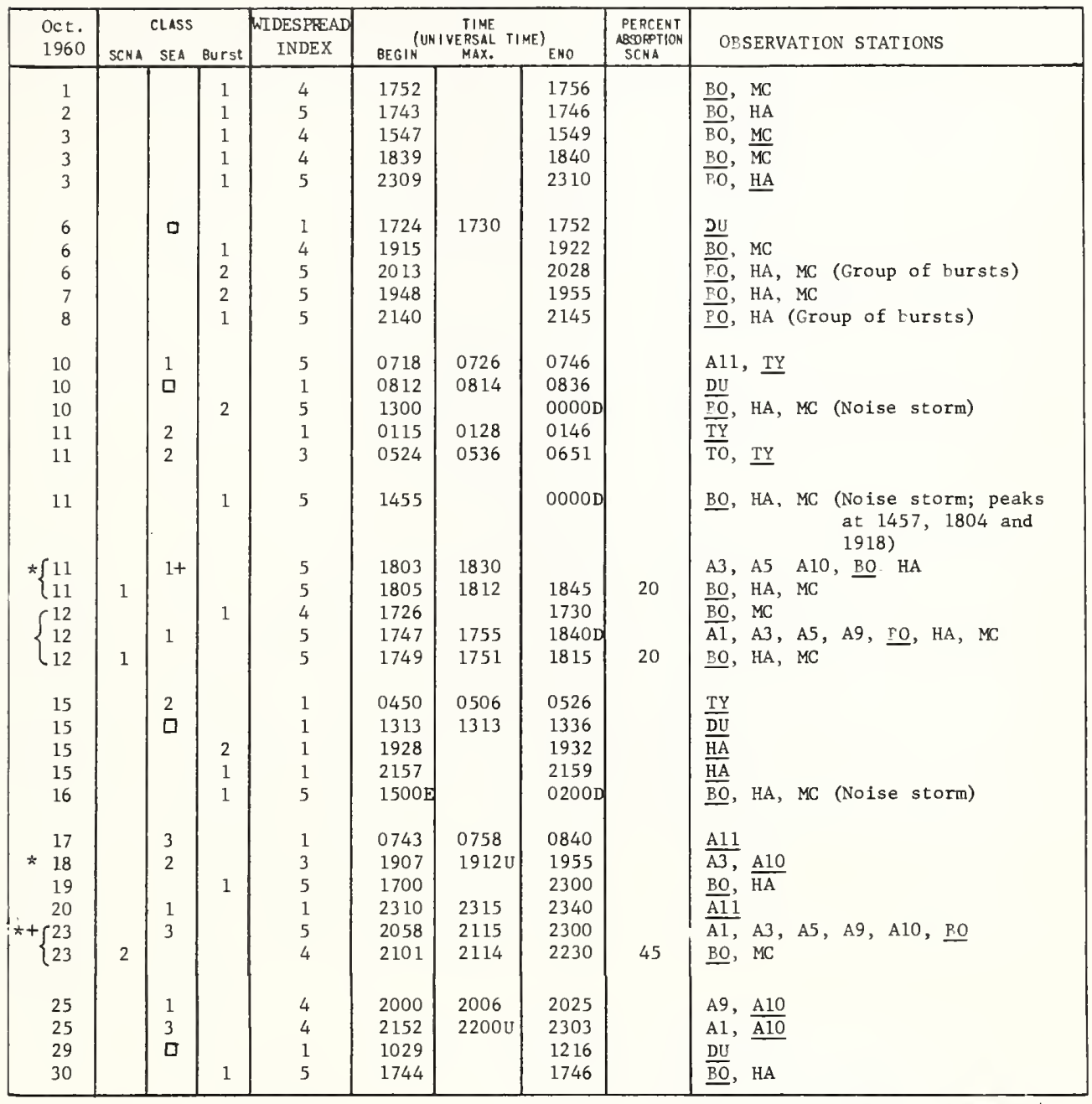

COMERECE - STAMOAROS - DUULDER

* = Sudden Enhancement of Signal from $18 \mathrm{kc}$ (NBA - Panama Canal Zone) observed by A5.

$+=$ Sudden Phase Anomaly of $18 \mathrm{kc}$ (NPA) at Boulder, Colorado. (Equipment

working October $14,15,21-25)$.

Note: No usable record at Sacramento Peak for October 1960.

Addenda to table published CRPL-F 194 Part B, October 1960

Station A2 should be added to the SEA reported August 6, 1960 at 1309 UT, 1508 UT and 1622 UT; August 11 at 1925 UT; August 16 at 2307 UT; August 19 at 1837 UT; August 25 at 1923 UT; and August 26 at 1426 UT. 
SOLAR RADIO EMISSION

\section{OUTSTANDING OGGURRENCES}

NOVEMBER 1960

OTTAWA ,

$2800 \mathrm{MC}$

\begin{tabular}{|c|c|c|c|c|c|c|c|}
\hline \multirow{2}{*}{$\begin{array}{l}\text { Nov. } \\
1960\end{array}$} & \multirow{2}{*}{\multicolumn{2}{|c|}{ Tyoe* }} & \multirow[t]{2}{*}{ Start UT } & \multirow{2}{*}{$\begin{array}{l}\text { Duration } \\
\text { HIs:Mins }\end{array}$} & \multicolumn{2}{|c|}{ Maxinuz } & \multirow[t]{2}{*}{ Ramarks } \\
\hline & & & & & Twe Ui & $\begin{array}{l}\text { Peak } \\
\text { Fiux }\end{array}$ & \\
\hline 2 & 3 & Simple 3 & 1740 & 50 & 1743 & 4 & \\
\hline 2 & 1 & Simple 1 & 1913 & 1 & 1913.5 & 4 & \\
\hline 2 & 6 & Comp lex & 1941 & 4 & 1942 & 3 & \\
\hline 3 & 6 & Comp lex & 1455 & 12 & 1456.5 & 4 & \\
\hline 3 & 6 & Complex f & 1821.5 & 10 & 1824 & 9 & \\
\hline 5 & 3 & Simple 3 & b 1240 & $>2$ & 1310 & 8 & \\
\hline 5 & 1 & Simple 1 & 1555.7 & 1 & 1556 & 5 & \\
\hline 5 & 3 & Simple 3 & 1845 & 15 & 1851 & 2 & \\
\hline 6 & & Simple 3 f A & 1628 & $>4$ & 1900 & 28 & \\
\hline & 2 & Simple 2 f & 1835 & 25 & 1838 & 28 & \\
\hline 7 & 1 & Simple 1 & 1512 & 2 & 1512.8 & 7 & \\
\hline 8 & 3 & Simple $3 \mathrm{~A}$ & 1345 & 00 & 1455 & 17 & \\
\hline & 1 & Simple 1 & 1426 & 4 & 1428.2 & 6 & \\
\hline 10 & 2 & Simple 2 & 1430 & 10 & 1433.5 & 11 & \\
\hline 11 & 6 & Complex $\mathbf{f}$ & 1447 & 10 & 1450 & 10 & \\
\hline 11 & 1 & Simple 1 & 1508.5 & 1 & 1509 & 6 & \\
\hline 12 & 9 & Precursor & b 1240 & $>40$ & & 11 & \\
\hline & & Great Burst & 1320 & 40 & 1345.5 & 5500 & \\
\hline 13 & 1 & Simple 1 & 1732.5 & 5 & 1734.3 & 5 & \\
\hline 13 & 1 & Simple 1 & 1859 & 2 & 1900 & 3 & \\
\hline 17 & 6 & Comp lex & 1504.5 & 4 & 1506 & 38 & \\
\hline & 4 & Post Increase & & 15 & & 5 & \\
\hline 19 & .1 & Simple 1 & 1356.3 & 1.5 & 1356.6 & 6 & \\
\hline 19 & 1 & Simple 1 & 1554 & 2 & 1555 & 4 & \\
\hline 19 & 3 & Simple $3 \mathrm{~A}$ & 1740 & 00 & 1732 & 6 & \\
\hline & 1 & Simple 1 & 1744.7 & 2 & 1745.3 & 4 & \\
\hline 20 & 9 & Precursor $\mathrm{f}$ & 1939 & 44 & & 14 & \\
\hline & 6 & Complex $\mathbf{f}$ & 2023 & $>47$ & 2026.5 & 400 & In sunset osc. \\
\hline 26 & 3 & Simple 3 & 1542 & $>4$ & indet. & 4 & \\
\hline 28 & 3 & Simple $3 \mathrm{~A}$ & 1600 & $>4$ & indet. & 4 & \\
\hline & 1 & Simple 1 & 1610.2 & 2 & 1611 & 6 & \\
\hline 30 & 2 & Simple 2 & 2012 & 1 & 2012.3 & 9 & \\
\hline
\end{tabular}




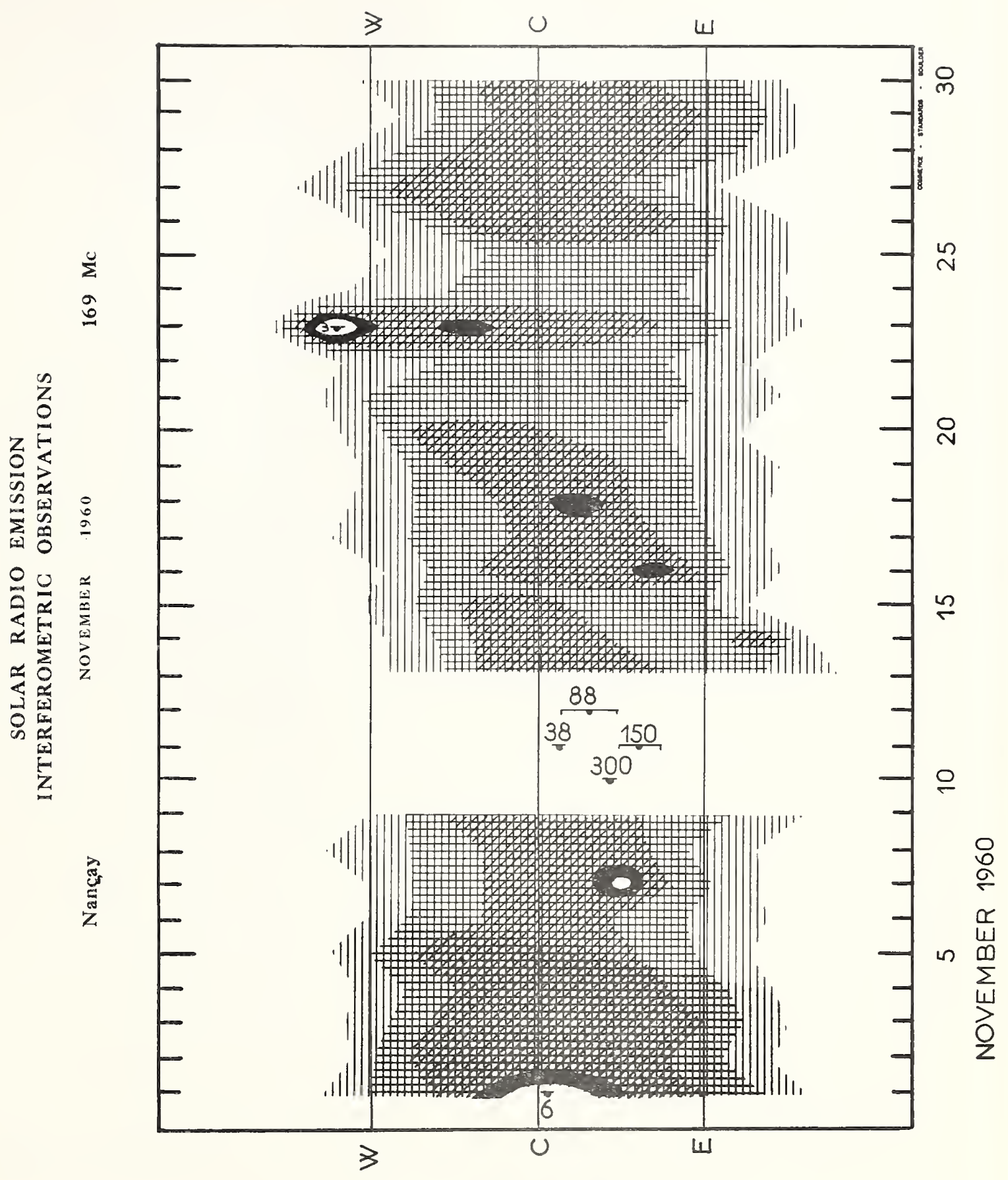




\begin{tabular}{|c|c|c|c|c|c|}
\hline $\begin{array}{l}\text { Nov. } \\
1960\end{array}$ & Type & $\begin{array}{c}\text { Start } \\
\text { UT }\end{array}$ & $\begin{array}{c}\text { Time of } \\
\text { Maximum } \\
\text { UT }\end{array}$ & $\begin{array}{l}\text { Duration } \\
\text { Minutes }\end{array}$ & Intensity \\
\hline 1 & 6 & $1334 \mathrm{E}$ & & $216 \mathrm{D}$ & 1 \\
\hline 1 & 6 & $1750 \quad E$ & & $340 \quad D$ & 2 \\
\hline 2 & 3 & 1747.6 & 1747.6 & 0.3 & 2 \\
\hline 2 & 3 & 1813.4 & 1813.4 & 0.3 & 2 \\
\hline 2 & 3 & 2209.4 & 2209.4 & 0.3 & 2 \\
\hline 3 & 3 & 1512.1 & 1512.1 & 0.4 & 2 \\
\hline 3 & 3 & 1625.0 & 1625.1 & 0.3 & 2 \\
\hline 3 & 3 & 2308.8 & 2308.9 & 0.2 & 2 \\
\hline 4 & 3 & 1337.6 & 1338.0 & 0.4 & 2 \\
\hline 4 & 3 & 1421.5 & 1422.1 & 0.6 & 2 \\
\hline 4 & 3 & 1423.5 & 1424.0 & 0.5 & 2 \\
\hline 4 & 3 & 2022.4 & 2022.8 & 1.0 & 2 \\
\hline 5 & 3 & 2131.5 & 2132.5 & 1.1 & 2 \\
\hline 5 & 2 & 2154.2 & 2155.5 & 4.8 & 1 \\
\hline 5 & 3 & 2314.1 & 2314.5 & 0.2 & 2 \\
\hline 6 & 3 & 1605.5 & 1606.1 & 0.4 & 2 \\
\hline 6 & 3 & 1609.5 & 1609.6 & 0.5 & 1 \\
\hline 6 & 2 & 1647.5 & 1649.6 & 2.1 & 2 \\
\hline 6 & 9 & 1827.0 & 1839.5 & 31 & 1 \\
\hline 6 & 8 & 1900.0 & 1904.0 & 8 & 1 \\
\hline 6 & 3 & 1906.3 & 1907.1 & 1.2 & 3 \\
\hline 6 & 3 & 1951.0 & 1951.4 & 0.4 & 2 \\
\hline 6 & 3 & 1957.9 & 1958.3 & 0.4 & 2 \\
\hline 6 & 7 & 2115 & & $131 \mathrm{D}$ & 1 \\
\hline 6 & 3 & 2139.0 & 2140.0 & 1.1 & 3 \\
\hline 7 & 3 & 1422.2 & 1422.3 & 1.0 & 2 \\
\hline 7 & 3 & 1440.3 & 1440.5 & 0.4 & 2 \\
\hline 7 & 2 & 2050.9 & 2053.0 & 4.3 & 3 \\
\hline 7 & 8 & 2130.4 & 2132.5 & 3.9 & 3 \\
\hline 8 & 3 & 2237.2 & 2237.5 & 0.4 & 3 \\
\hline 9 & 3 & 1411.0 & 1411.2 & 0.2 & 2 \\
\hline 9 & 3 & 1445.0 & 1446.0 & 1.0 & 3 \\
\hline 9 & 2 & 1749.0 & 1800.8 & 20 & 1 \\
\hline 9 & 1 & 2232.5 & 2301.8 & 49 D & 2 \\
\hline 10 & 6 & $1344 \mathrm{E}$ & 1526 & $578 \quad \mathrm{D}$ & 2 \\
\hline 11 & 6 & $1345 \mathrm{E}$ & & 576 D & 3 \\
\hline 12 & 6 & 1347 & & $573 \mathrm{D}$ & 2 \\
\hline 13 & 3 & 1510.0 & 1511.0 & 2.0 & 1 \\
\hline 13 & 3 & 1632.5 & 1632.8 & 0.3 & 2 \\
\hline 13 & 3 & 1643.2 & 1643.3 & 0.3 & 2 \\
\hline 13 & 3 & 1711.5 & 1711.6 & 0.3 & 1 \\
\hline 13 & 3 & 1726.5 & 1726.6 & 1.0 & 2 \\
\hline 13 & 3 & 1815.0 & 1815.3 & 1.1 & 1 \\
\hline 13 & 3 & 1931.6 & 1932.1 & 1.0 & 2 \\
\hline 14 & 3 & 1349.9 & 1350.0 & 0.3 & 2 \\
\hline 14 & 3 & 1436.8 & 1437.1 & 0.3 & 2 \\
\hline 14 & 3 & 1746.0 & 1746.1 & 0.5 & 2 \\
\hline 14 & 3 & 1758.1 & 1758.9 & 0.8 & 2 \\
\hline 14 & 3 & 2253.8 & 2253.9 & 0.3 & 3 \\
\hline 14 & 3 & 2302.5 & 2302.6 & 1.1 & 2 \\
\hline 15 & 3 & 1400.7 & 1401.0 & 0.3 & 3 \\
\hline 15 & 3 & 1553.1 & 1553.2 & 0.2 & 2 \\
\hline 15 & 3 & 1619.0 & 1619.3 & 0.4 & 2 \\
\hline 15 & 3 & 1727.5 & 1728.0 & 0.7 & 3 \\
\hline 15 & 3 & 1728.5 & 1728.7 & 0.7 & 2 \\
\hline
\end{tabular}

\begin{tabular}{|c|c|c|c|c|c|}
\hline $\begin{array}{l}\text { Nov. } \\
1960\end{array}$ & Type & $\begin{array}{c}\text { Start } \\
\text { UT }\end{array}$ & $\begin{array}{c}\text { Time of } \\
\text { Maximum } \\
\text { UT }\end{array}$ & $\begin{array}{c}\text { Duration } \\
\text { Minutes }\end{array}$ & Intensity \\
\hline 16 & 3 & 1755.2 & 1756.0 & 1.0 & 2 \\
\hline 16 & 3 & 2041.6 & 2041.7 & 0.2 & 2 \\
\hline 16 & 3 & 2138.8 & 2138.9 & 0.2 & 2 \\
\hline 16 & 3 & 2237.3 & 2237.7 & 1.2 & 2 \\
\hline 16 & 3 & 2243.5 & 2243.6 & 0.2 & 2 \\
\hline 16 & 3 & 2247.5 & 2248.1 & 1.5 & 2 \\
\hline 17 & 3 & 1431.1 & 1431.6 & 0.5 & 2 \\
\hline 17 & 3 & 1455.7 & 1456.4 & 0.8 & 2 \\
\hline 17 & 2 & 1647.0 & 1647.7 & 1.4 & 1 \\
\hline 17 & 3 & 2115.4 & 2115.4 & 0.3 & 2 \\
\hline 17 & 3 & 2133.8 & 2134.8 & 1.2 & 2 \\
\hline 17 & 3 & 2310.4 & 2310.4 & 0.5 & 2 \\
\hline 18 & 3 & 2023.3 & 2024.1 & 1.2 & 2 \\
\hline 18 & 3 & 2246.8 & 2246.9 & 0.2 & 2 \\
\hline 19 & 3 & 1513.5 & 1513.8 & 0.7 & 2 \\
\hline 19 & 3 & 1557.5 & 1557.5 & 1.0 & 2 \\
\hline 19 & 3 & 1600.0 & 1600.4 & 1.0 & 2 \\
\hline 19 & 3 & 1624.6 & 1625.0 & 2.0 & 2 \\
\hline 19 & 3 & 1649.0 & 1650.5 & 3.0 & 1 \\
\hline 19 & 8 & 1658.2 & 1658.3 & 4.8 & 3 \\
\hline 19 & 2 & 1741.2 & 1749.5 & 9 & 2 \\
\hline 19 & 2 & 1956.2 & 1958.7 & 18 & 1 \\
\hline 19 & 3 & 2156.1 & 2156.3 & 0.3 & 2 \\
\hline 20 & $9 \mathrm{~A}$ & 2027.5 & 2033.0 & 7 & 3 \\
\hline 20 & $9 B$ & 2038.0 & 2039.9 & 15 & 3 \\
\hline 20 & 8 & 2135.4 & 2137.5 & 3 & 1 \\
\hline 20 & 3 & 2255.4 & 2255.5 & 1.1 & 2 \\
\hline 21 & 3 & 1402.0 & 1402.5 & 0.4 & 2 \\
\hline 21 & 3 & 1656.0 & 1656.1 & 0.5 & 2 \\
\hline 21 & 3 & 2134.6 & 2135.0 & 0.5 & 2 \\
\hline 21 & 3 & 2155.1 & 2155.5 & 0.4 & 2 \\
\hline 21 & 3 & 2202.6 & 2202.9 & 0.4 & 2 \\
\hline 21 & 3 & 2245.5 & 2245.9 & 0.4 & 2 \\
\hline 21 & 3 & 2254.4 & 2254.9 & 0.5 & 2 \\
\hline 22 & 3 & 1405.6 & 1406.1 & 0.5 & 2 \\
\hline 22 & 7 & 1934 & & $213 \mathrm{D}$ & 1 \\
\hline 23 & 3 & 1417.6 & 1417.7 & 0.9 & 2 \\
\hline 23 & 2 & 1425.5 & 1427.5 & 2.5 & 2 \\
\hline 23 & 3 & 1435.3 & 1435.7 & 0.4 & 2 \\
\hline 23 & 7 & 1505 & 1557 & 90 & 1 \\
\hline 23 & 8 & 1629.0 & 1630.2 & 6.4 & 3 \\
\hline 23 & 2 & 1819.3 & 1827.8 & 20 & 2 \\
\hline 23 & 3 & 2053.0 & 2053.3 & 1.0 & 2 \\
\hline 23 & 3 & 2211.0 & 2211.6 & 0.4 & 2 \\
\hline 24 & 3 & 2048.0 & 2048.2 & 2.1 & 2 \\
\hline 25 & 3 & 2207.2 & 2207.5 & 0.3 & 2 \\
\hline 27 & 3 & 1843.9 & 1844.0 & 0.3 & 2 \\
\hline 27 & 3 & 2139.4 & 2139.5 & 0.2 & 2 \\
\hline 27 & 3 & 2238.8 & 2239.1 & 0.3 & 2 \\
\hline 28 & 3 & 2136.0 & 2136.4 & 1.0 & 2 \\
\hline 30 & 3 & 1611.0 & 1612.2 & 0.7 & 2 \\
\hline
\end{tabular}


TIMES OF OBSERVATIONS

BOULDER

$108 \mathrm{MC}$

\begin{tabular}{|c|c|c|c|c|c|c|}
\hline $\begin{array}{l}\text { Nov. } \\
1960\end{array}$ & U.T. & & $\begin{array}{l}\text { Nov. } \\
1960\end{array}$ & U.T. & & \\
\hline $\begin{array}{r}2 \\
3 \\
4 \\
5 \\
6 \\
7 \\
8 \\
9 \\
10 \\
11 \\
12 \\
13 \\
14 \\
15\end{array}$ & $\begin{array}{l}1334-1710 ; \\
1750-2330 \\
1335-2329 \\
1336-2329 \\
1337-2328 \\
1339-2327 \\
1340-2326 \\
1341-2323 \\
1342-2323 \\
1343-2322 \\
1344-2322 \\
1345-2321 \\
1347-2320 \\
1348-2319 \\
1349-2317 \\
1350-1637 \\
1655-2316 \\
1520-2317 \\
1352-2316 \\
1354-2314\end{array}$ & 更 & $\begin{array}{l}19 \\
20 \\
21 \\
22 \\
23 \\
24 \\
25\end{array}$ & $\begin{array}{l}1459-2313 \\
1735-2312 \\
1357-1657 \\
1713-2308 \\
1358-2307 \\
1359-2308 \\
1400-2308 \\
1401-2036 ; \\
2046-2230 ; \\
2247-2306 \\
1402-1706 ; \\
1714-1730 ; \\
2218-2305 \\
1404-2305 \\
1405-2200 ; \\
2213-2306 \\
1406-2304 \\
1407-2304\end{array}$ & $\begin{array}{l}\text { I } \\
\text { I }\end{array}$ & $\begin{array}{l}1930-2020 \\
1718-2145 \\
1621-1830 \\
1935-2003\end{array}$ \\
\hline
\end{tabular}


$\frac{5}{9}$
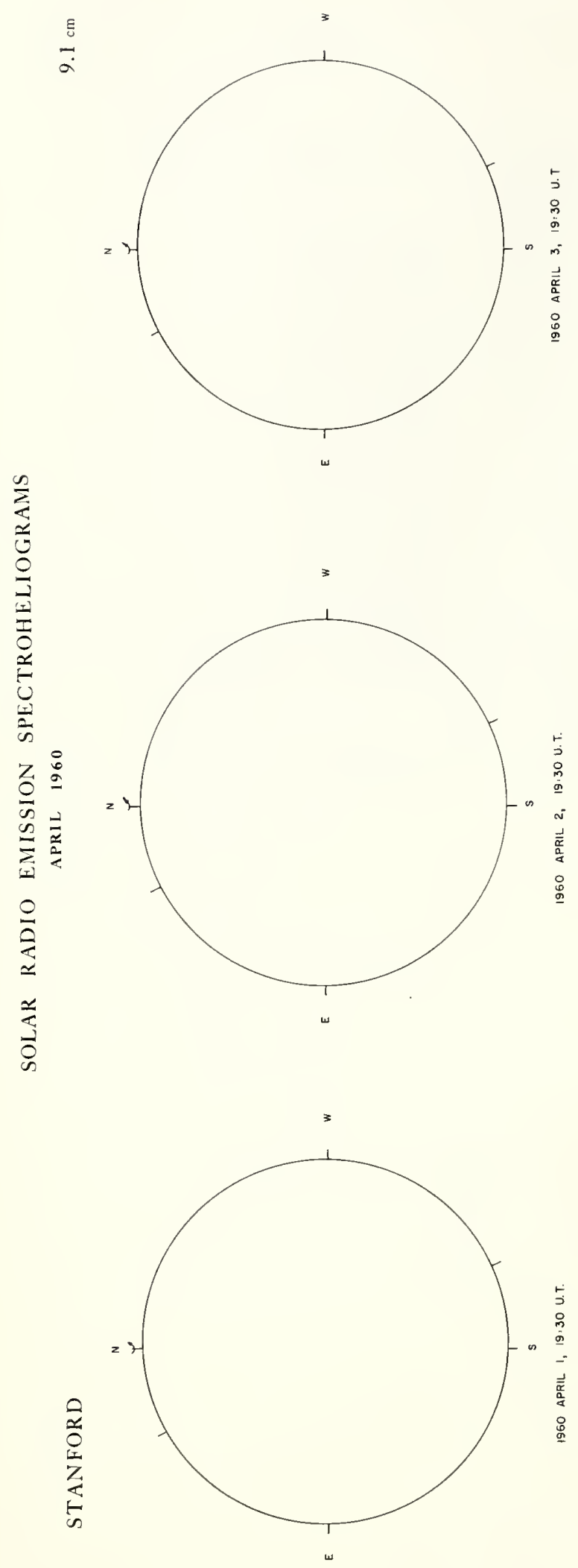
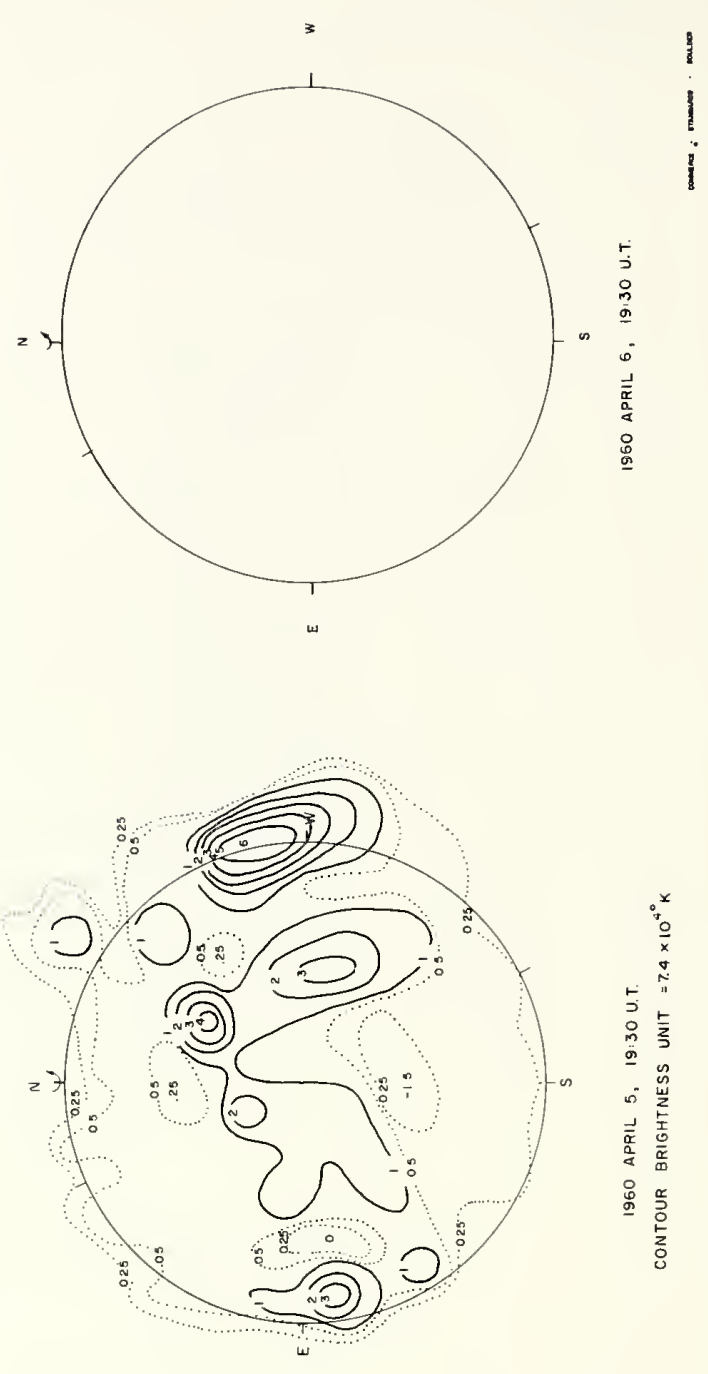

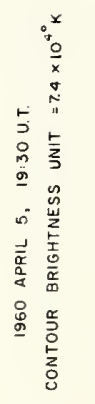

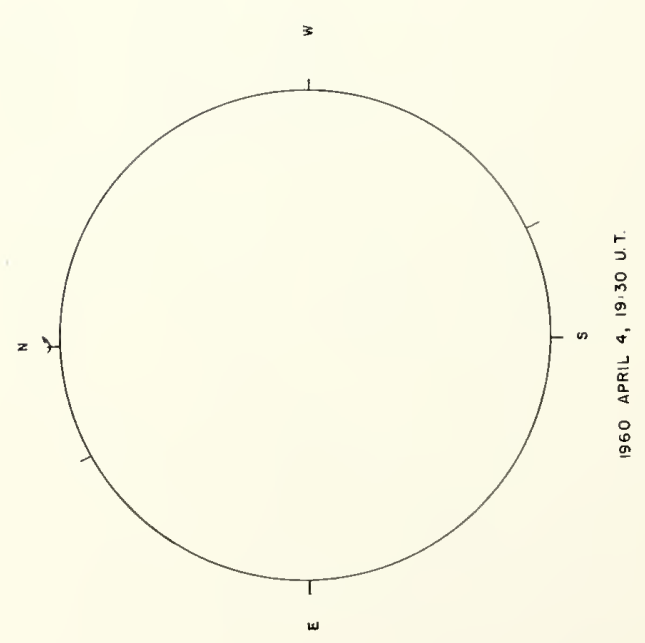


$\frac{5}{a}$

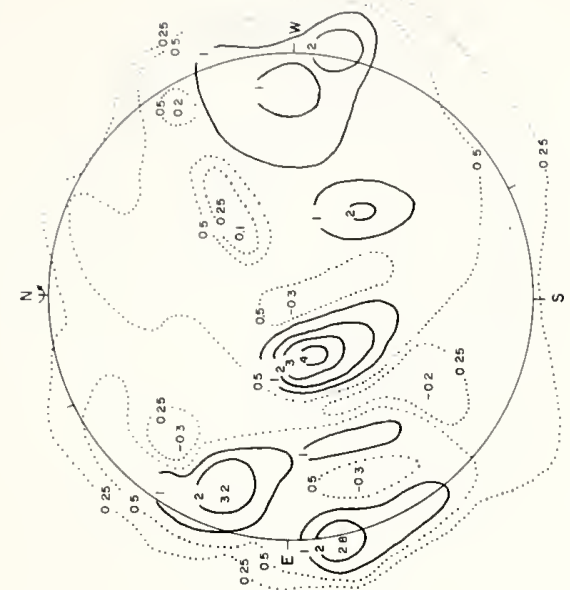



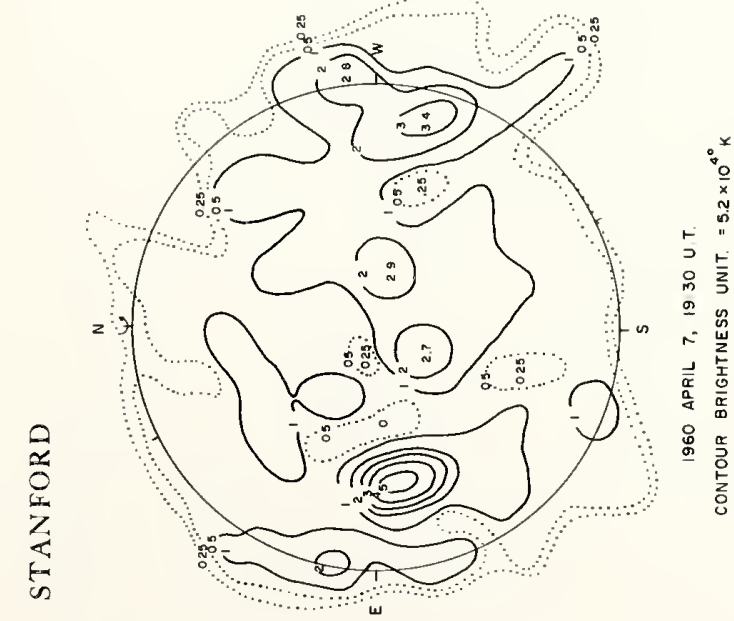

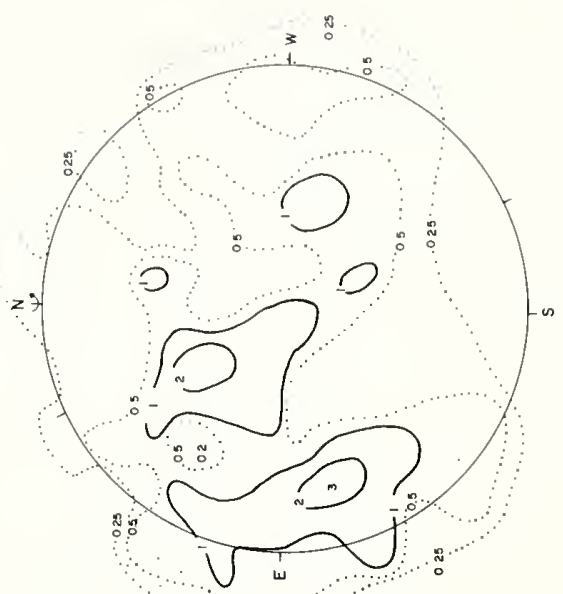
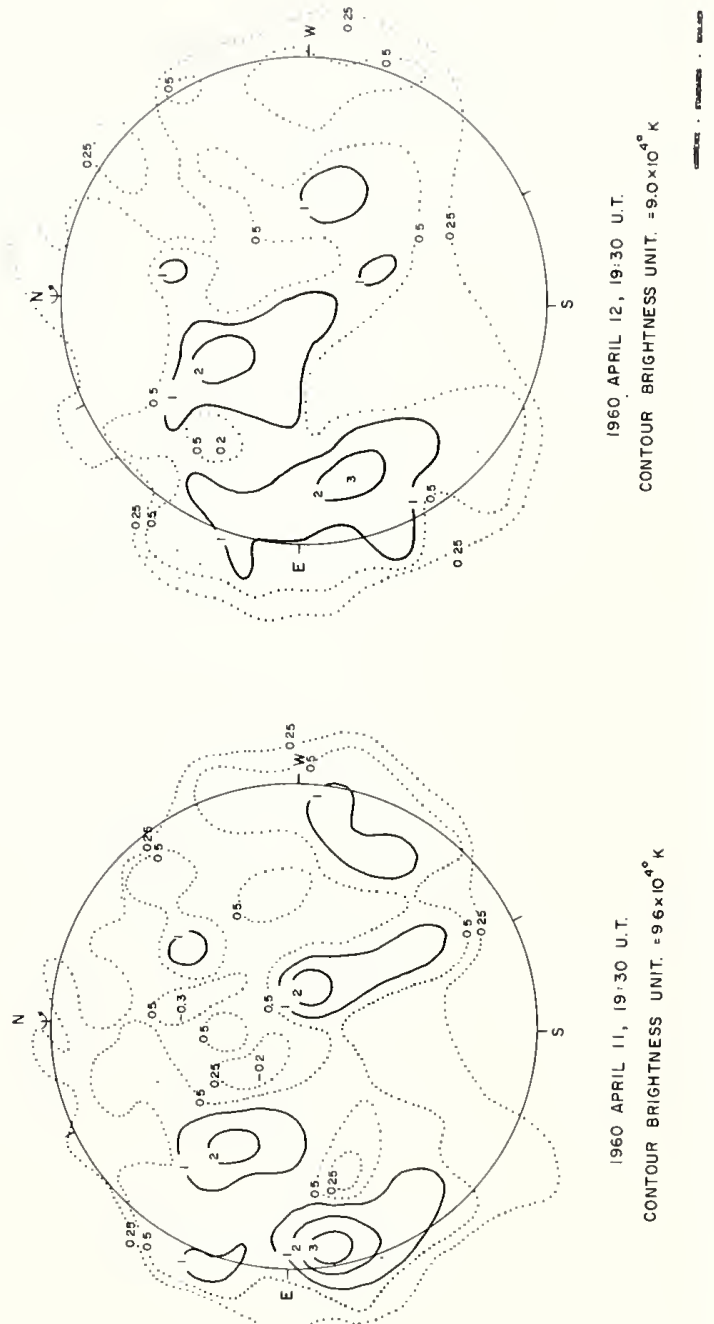

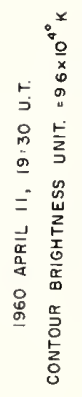

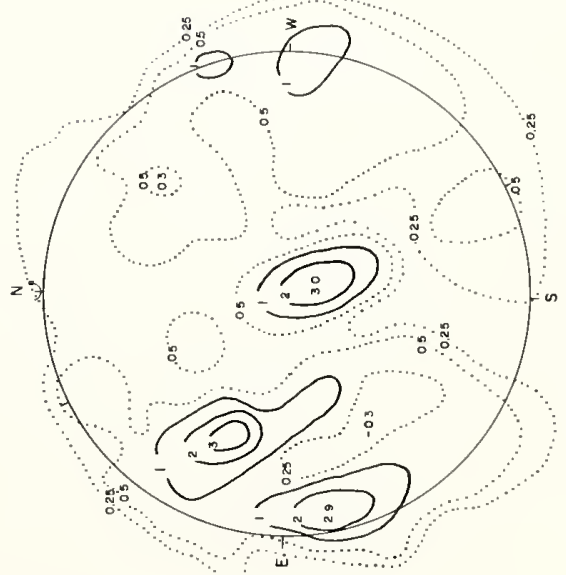

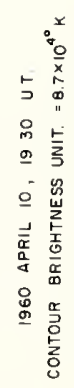


IVg

$\frac{5}{a}$
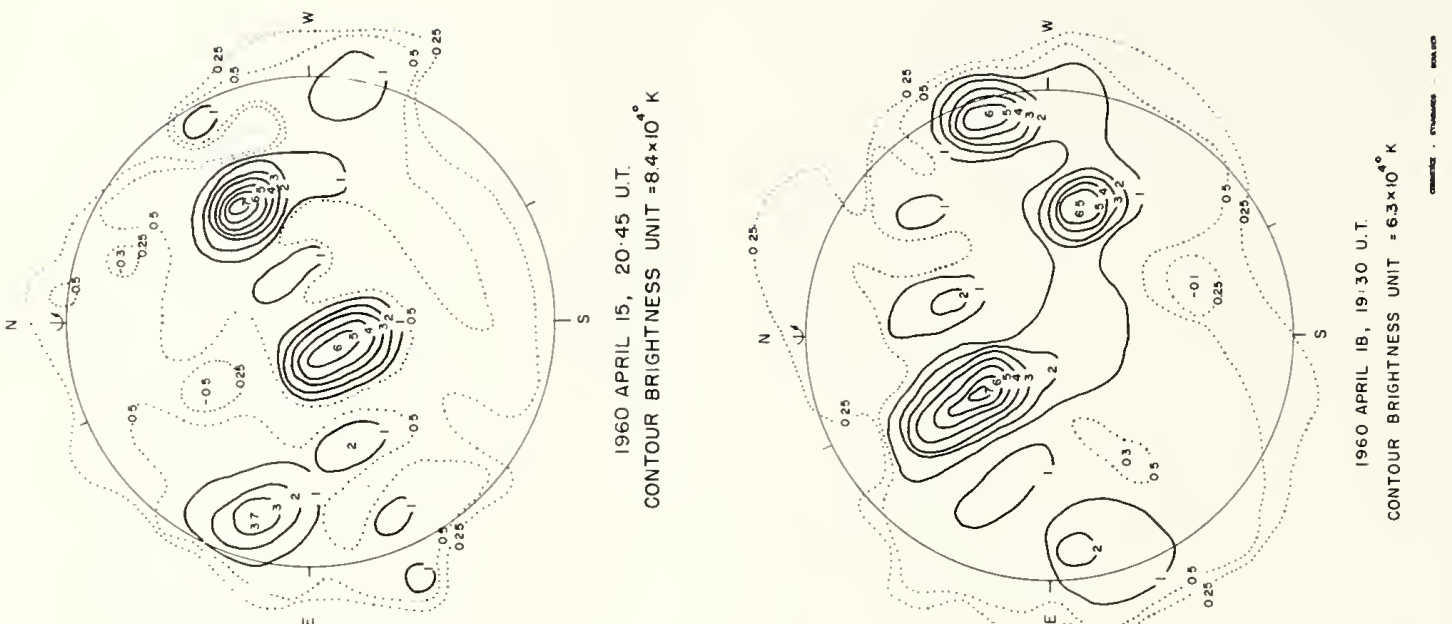

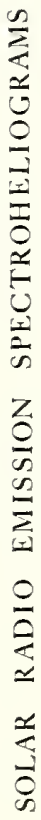
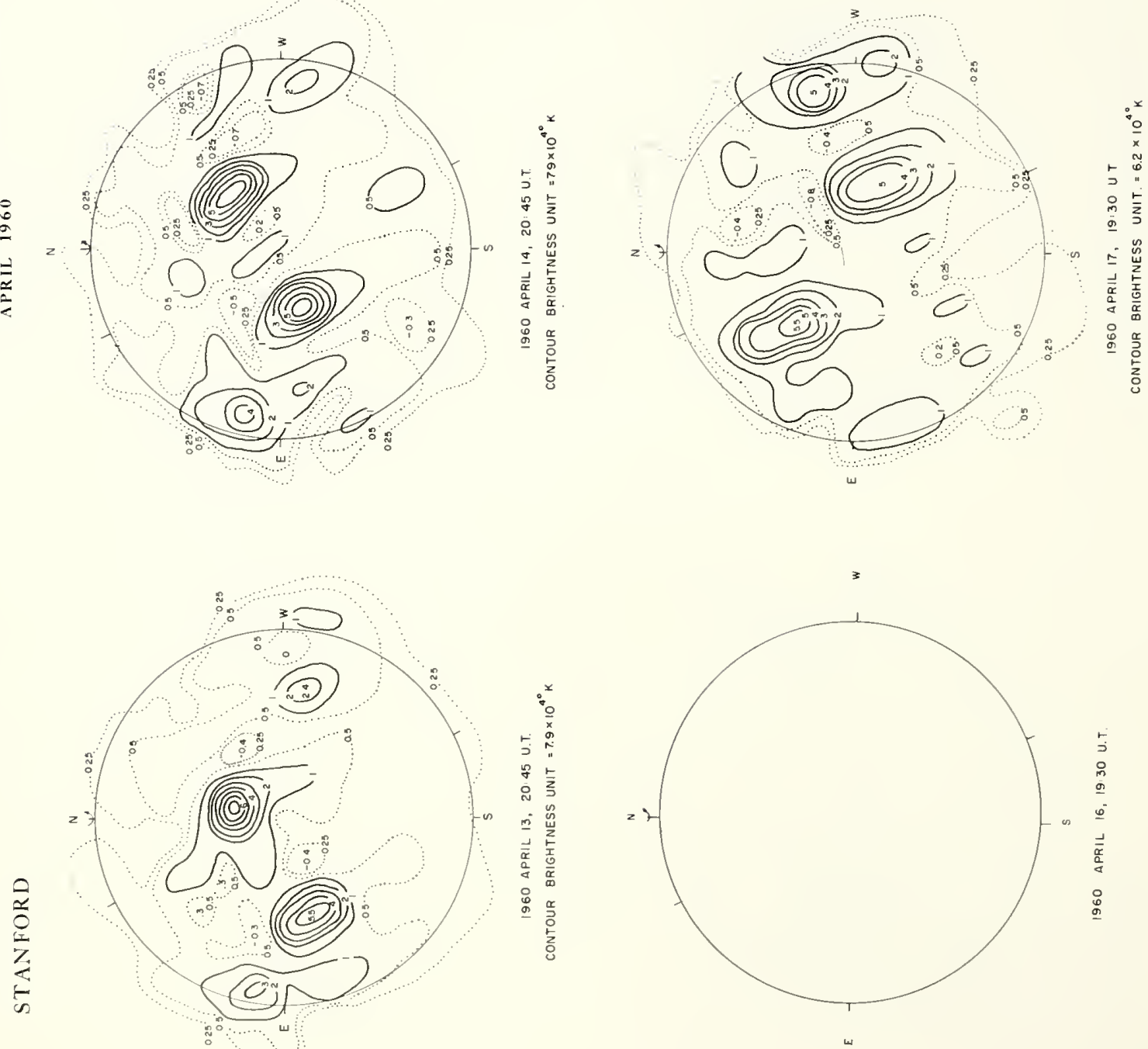

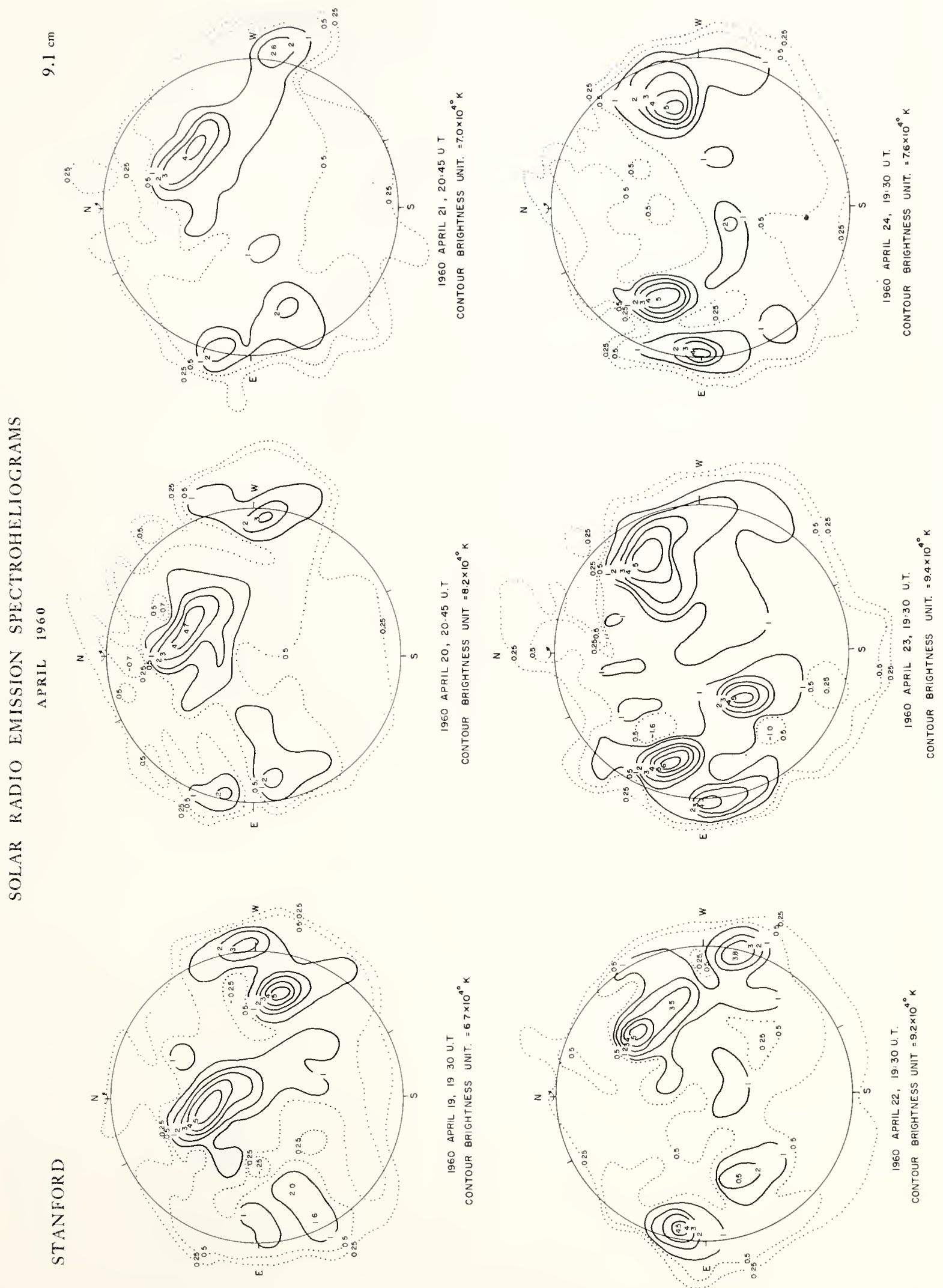
$\frac{5}{9}$
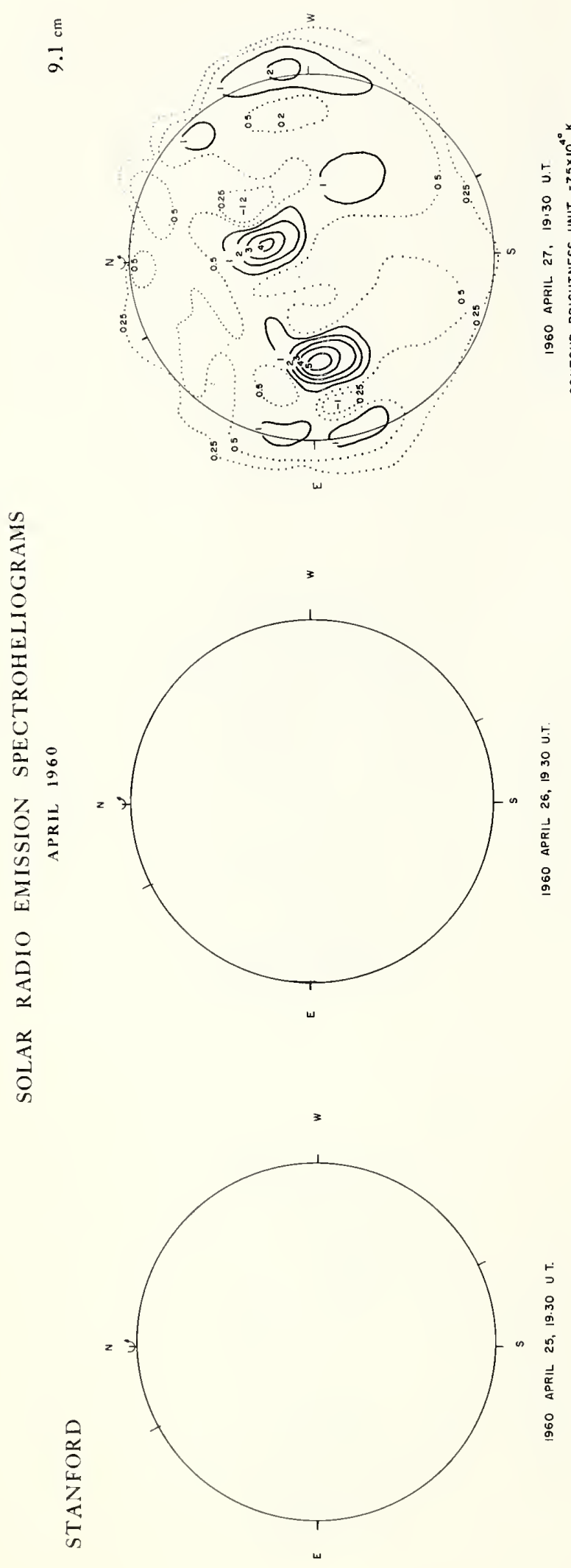
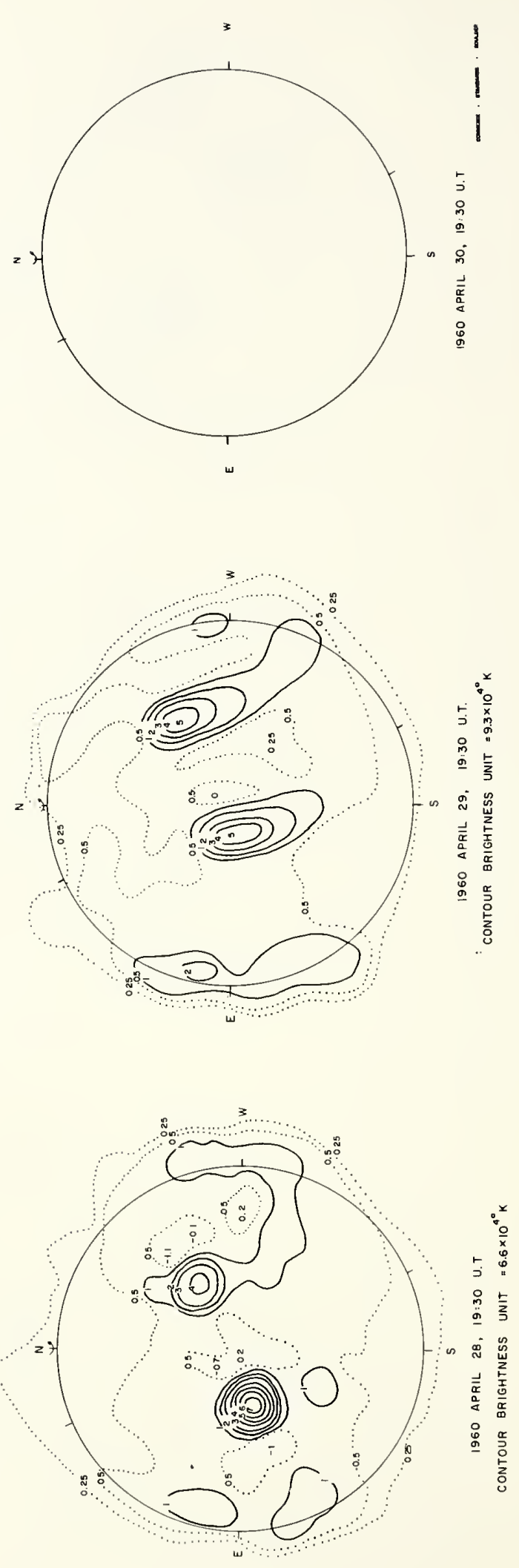

COSMIC RAY INDICES

(Climax Neutron Monitor)

\begin{tabular}{|r|c|c|c|}
\hline $\begin{array}{c}\text { Oct. } \\
1960\end{array}$ & $\begin{array}{c}\text { Daily } \\
\text { average } \\
\text { counts/hr }\end{array}$ & $\begin{array}{c}\text { Oct. } \\
1960\end{array}$ & $\begin{array}{c}\text { Daily } \\
\text { average } \\
\text { counts/hr }\end{array}$ \\
\hline 1 & 2909.3 & 17 & 2873.6 \\
2 & 2886.0 & 18 & 2898.3 \\
3 & 2898.2 & 19 & 2892.0 \\
4 & 2910.3 & 20 & 2889.2 \\
5 & 2888.4 & 21 & 2895.2 \\
6 & 2766.2 & 22 & 2891.3 \\
7 & 2786.7 & 23 & 2892.2 \\
8 & 2837.0 & 24 & 2882.0 \\
9 & 2858.8 & 25 & 2886.7 \\
10 & 2866.3 & 26 & 2896.4 \\
11 & 2880.1 & 27 & 2896.1 \\
12 & 2876.3 & 28 & 2907.7 \\
13 & 2863.8 & 29 & 2905.8 \\
14 & 2849.7 & 30 & 2895.0 \\
15 & 2839.1 & 31 & 2901.8 \\
16 & 2862.2 & & \\
\hline
\end{tabular}




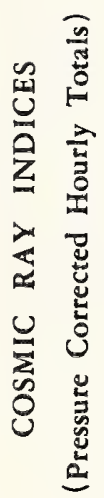

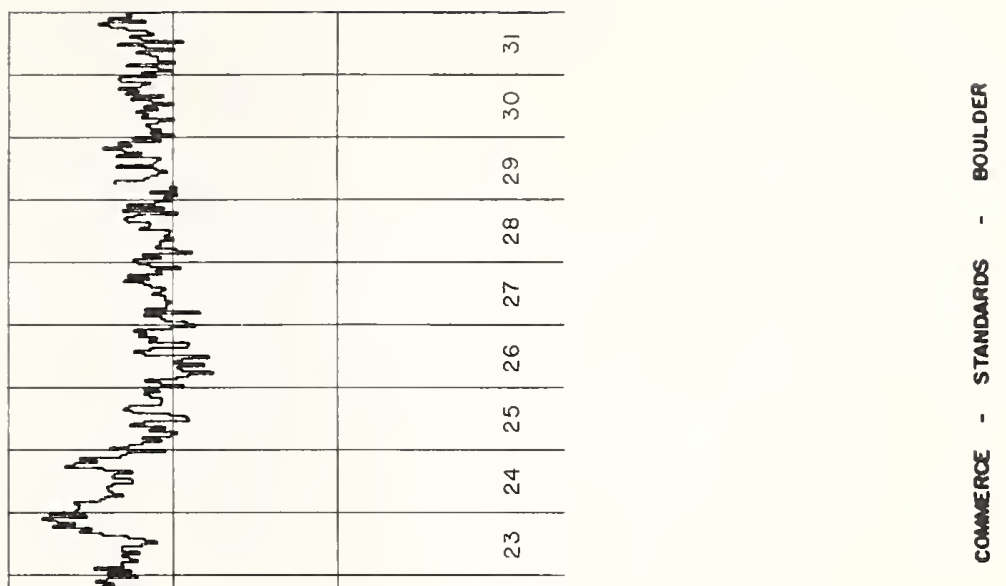


GEOMAGNETIC ACTIVITY INDICES

OCTOBER 1960

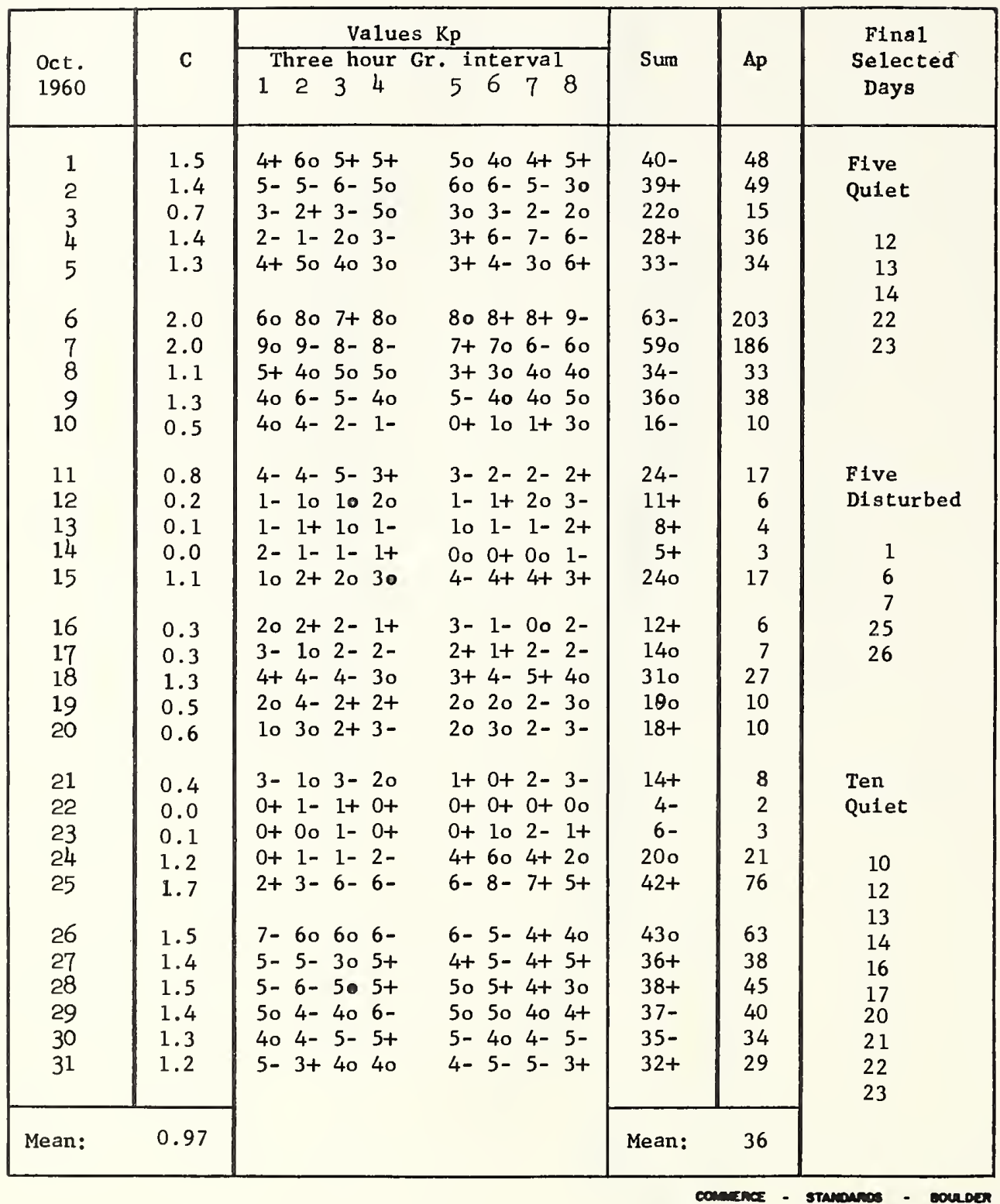




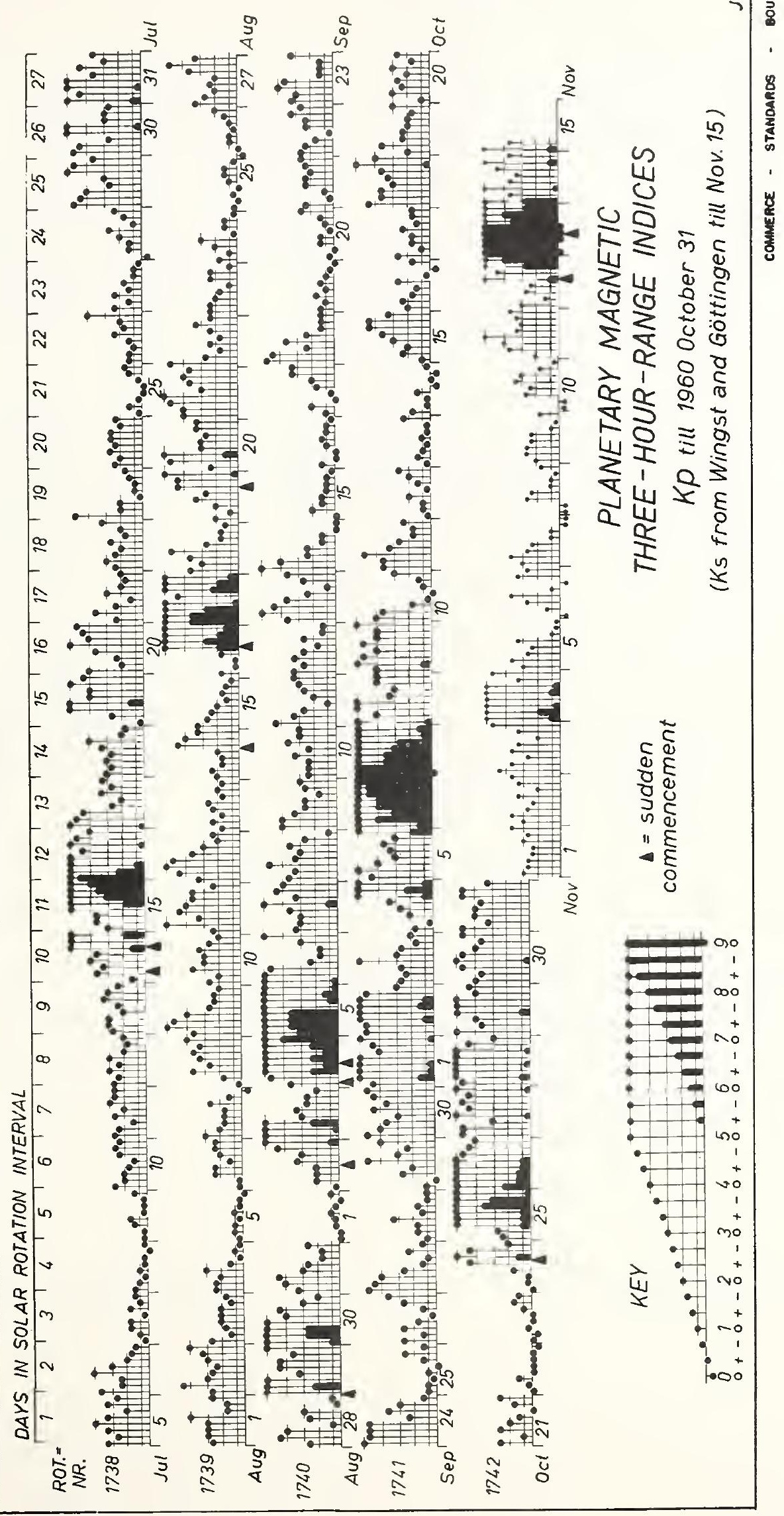


OCTOBER 1960

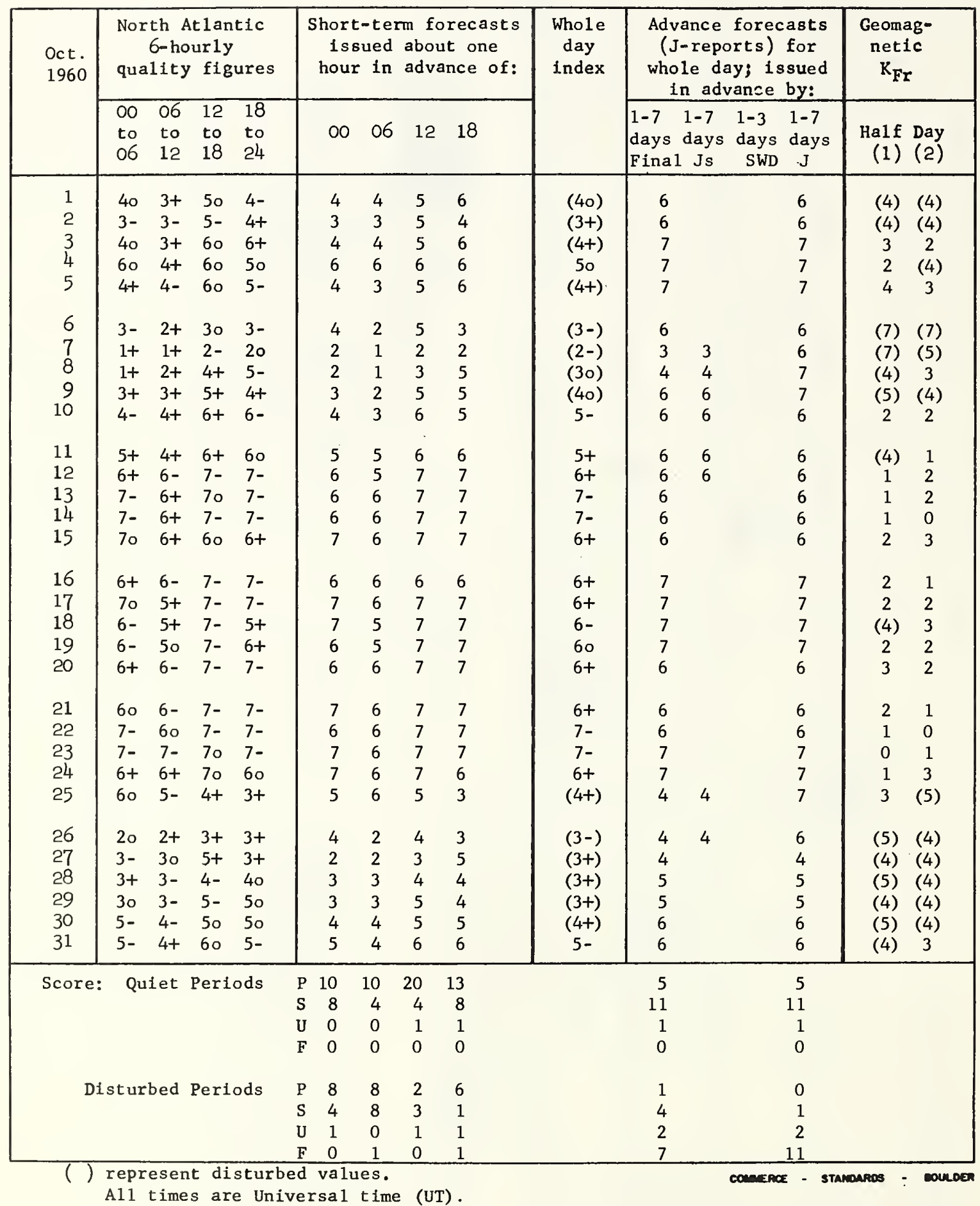


NORTH ATLANTIC

OCTOBER 1960

\section{- Short-term forecos $\uparrow$ \\ - Qualify figure}

$$
\text { I Range of reports }
$$
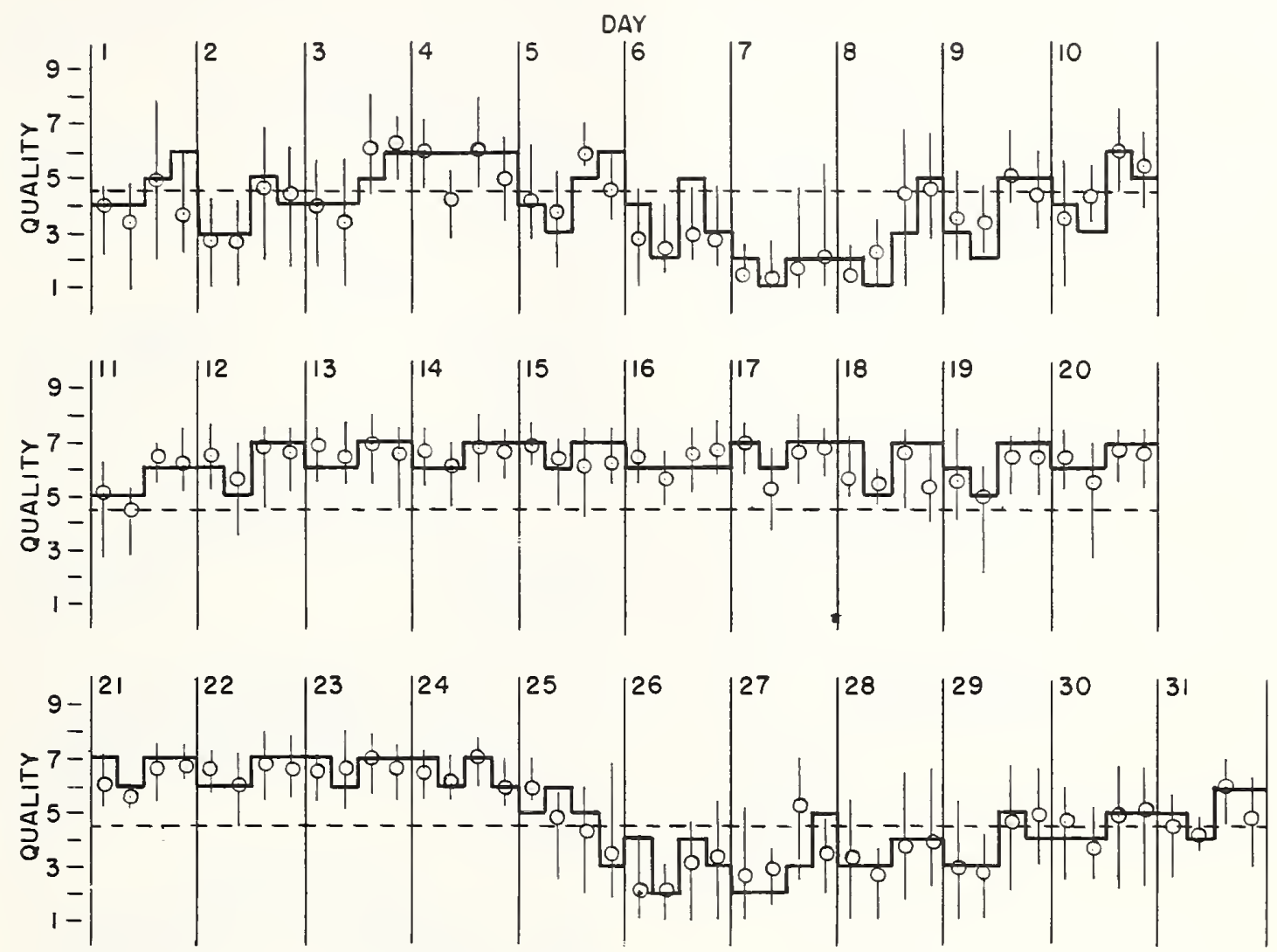

OUTCOME OF ADVANCED FORECASTS

FINAL ESTIMATE

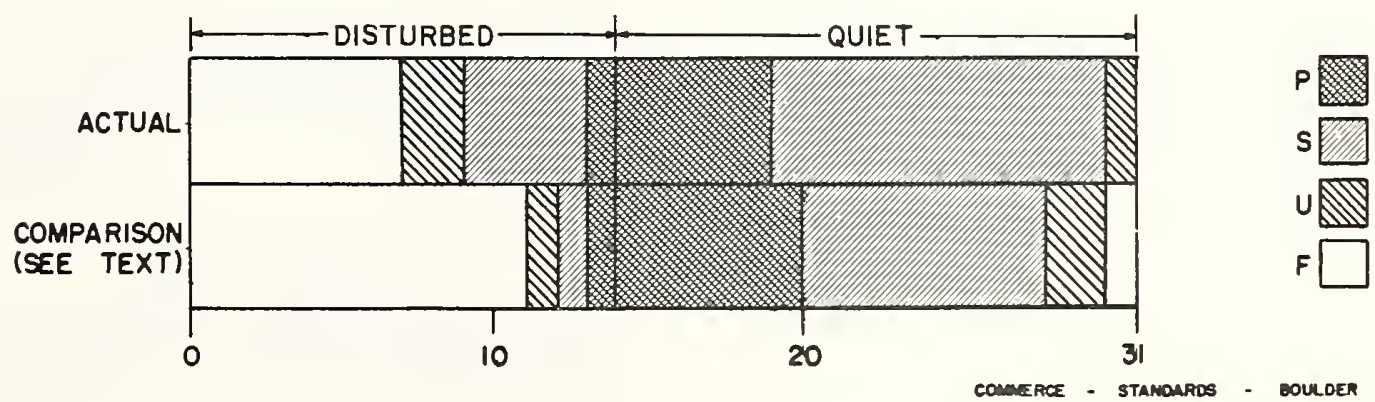



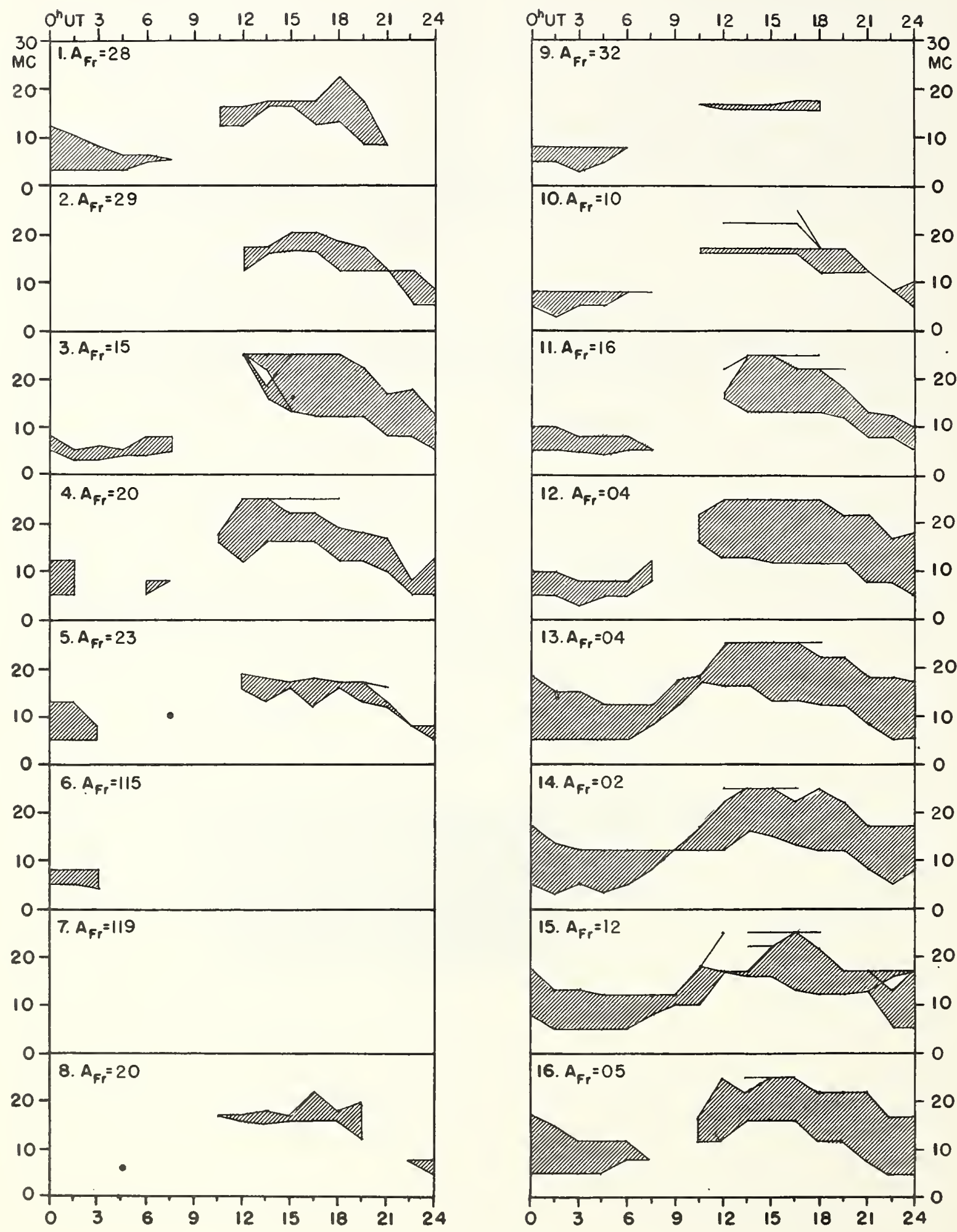

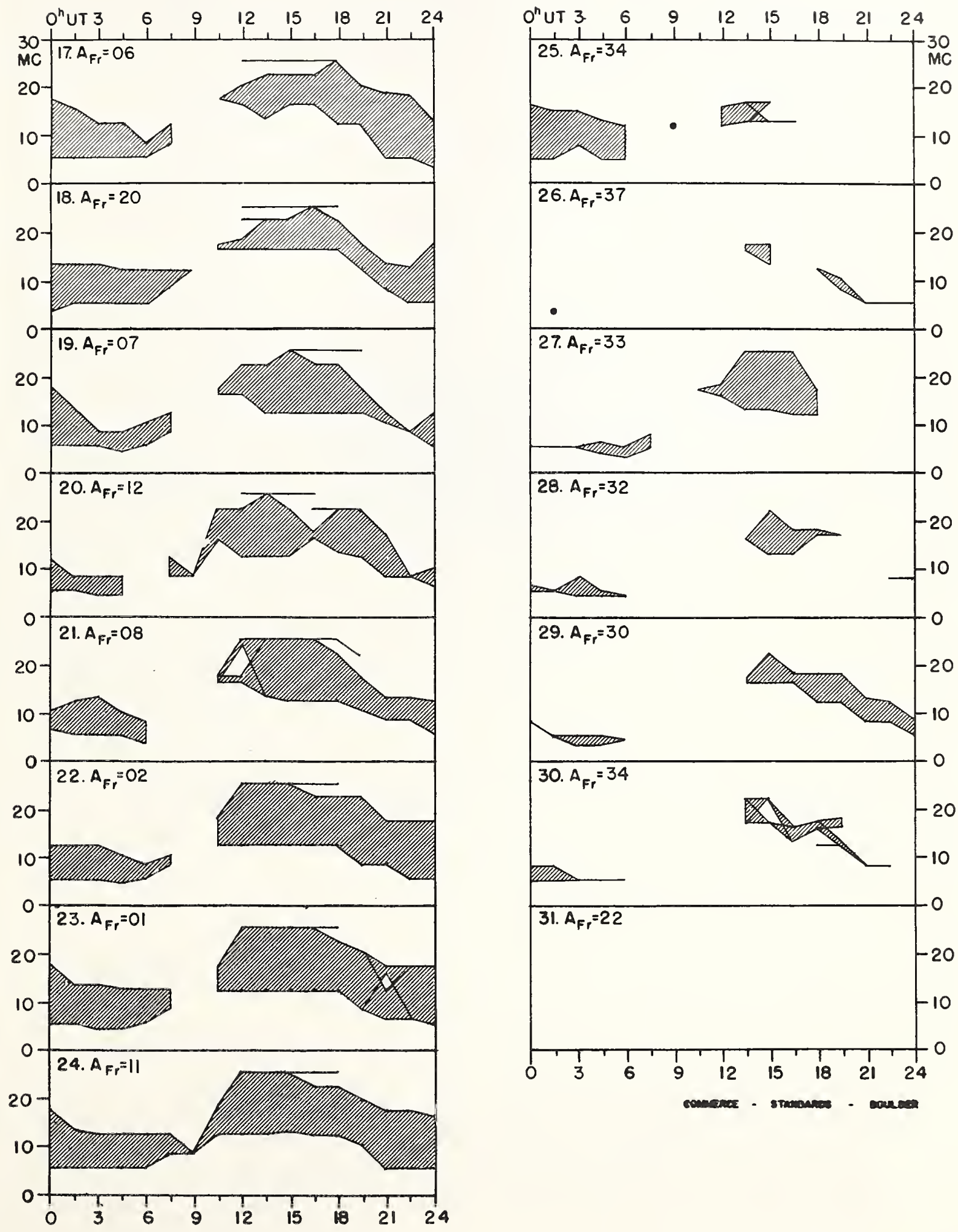
NORTH PACIFIC

OCTOBER 1960

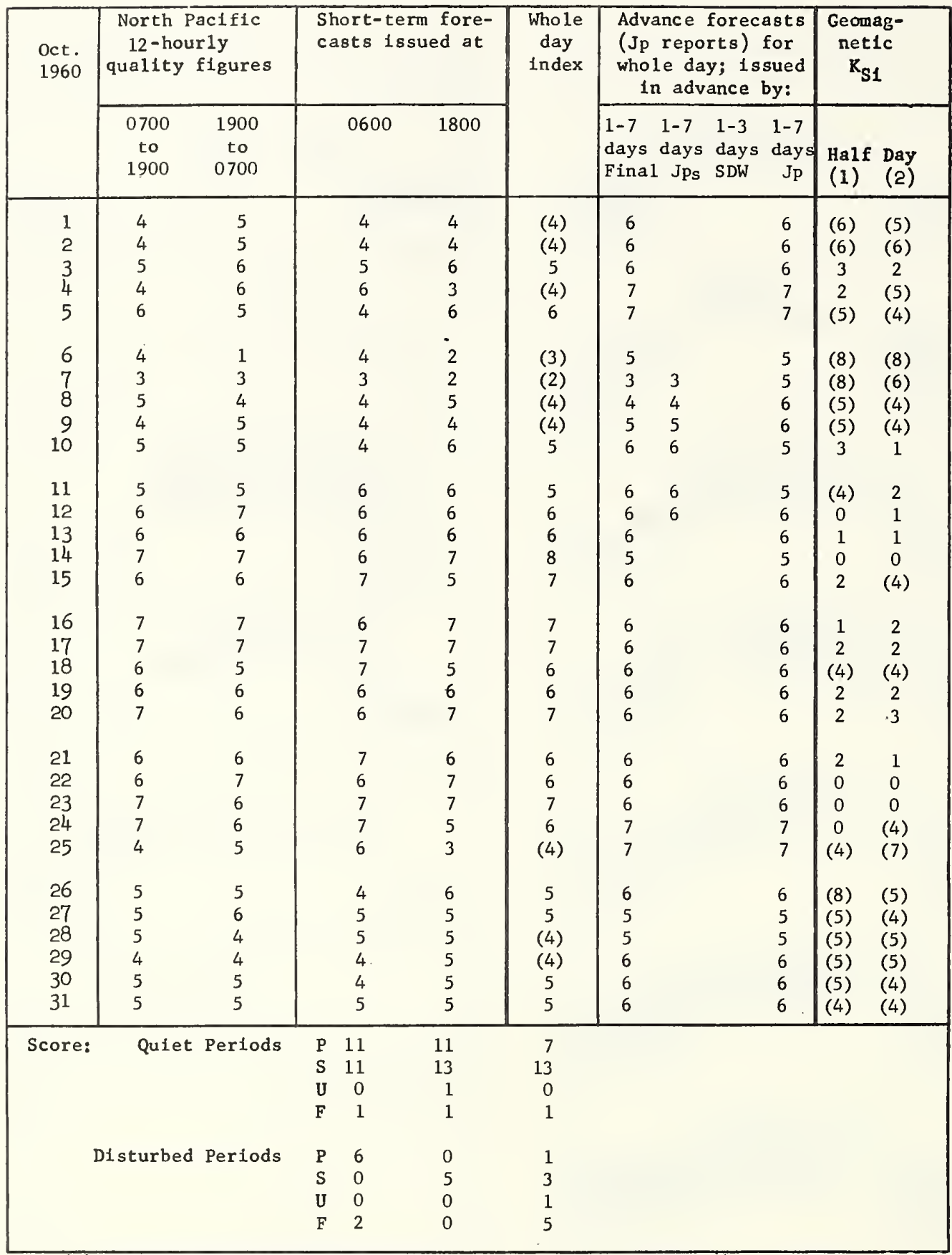

represent disturbed values. A1l times are Universal time (U.T.).

Note: From Oct. 25-30 the quality figures are not as disturbed as the magnetic indices suggest they should be. Blanketing sporadic-E was prevalent in the North Pacific area from Oct. 25 through Oct. 29, at the height of the magnetic storm. This could explain quality remaining fair. Vertical incidence data indicate lowered maximum usable frequencies but not complete blackout during the last week of October. 
NORTH PACIFIC

OC.TOBER 1960

OUTCOME OF ADVANCED FORECASTS FINAL ESTIMATE

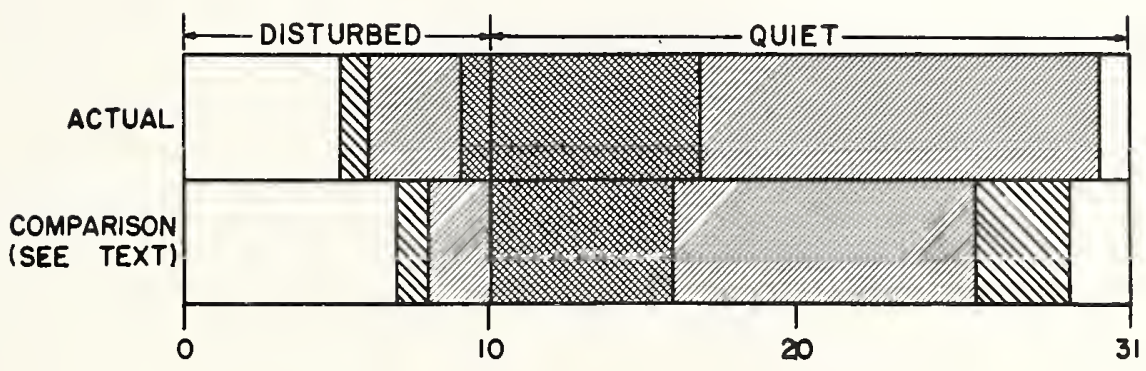

$P$ 奖

s

प जाए

$F$ 
INTERNATIONAL WORLD DAY SERVICE

NOVEMBER 1960

\begin{tabular}{|c|c|c|c|c|}
\hline $\begin{array}{c}\text { Issued } \\
\text { Day/Time UT } \\
\text { Nov. } \quad 1960\end{array}$ & Advance Geophysical Alert & No. & $\begin{array}{c}\text { World-wide Geophysical } \\
\text { Alert }\end{array}$ & Special Wor ld Interval \\
\hline $04 / 0250$ & Ft. Belvo1r, Magnetic Storm $03 / 22 \mathrm{XXz}$ & & & \\
\hline $04 / 1600$ & & 94 & Magnetic Storm $03 / 22 \mathrm{XX}$ & \\
\hline $12 / 1500$ & McMath, Solar Flare $12 / 13252$ & & & \\
\hline $12 / 1600$ & & 95 & Magnetic Storm $12 / 1350 z$ & Start Special World Interval \\
\hline $13 / 1600$ & & 96 & Cosmic Ray Increase $12 / 1345$ & Continue Special World Interval \\
\hline $14 / 1600$ & & 97 & & Finish Special World Interval \\
\hline $15 / 1330$ & Chicago, Cosmic Ray Increase $15 / 02 \mathrm{XXZ}$ & & & \\
\hline $15 / 1600$ & & 98 & $\begin{array}{l}\text { Cosmic Ray Increase } 15 / 02 \mathrm{XXZ} \\
\text { Magnetic Storm } 15 / 1305\end{array}$ & Start Special World Interval \\
\hline $16 / 1600$ & & 99 & & Continue Special World Interval \\
\hline $17 / 1600$ & & 100 & & Finish Special World Interval \\
\hline $21 / 1600$ & & 101 & Magnetic Storm 21/04XXZ & \\
\hline $25 / 0015$ & Ft. Belvoir, Magnetic Storm $24 / 20532$ & & & \\
\hline $25 / 1600$ & & 102 & Magnetic Storm 24/20532 & \\
\hline
\end{tabular}



